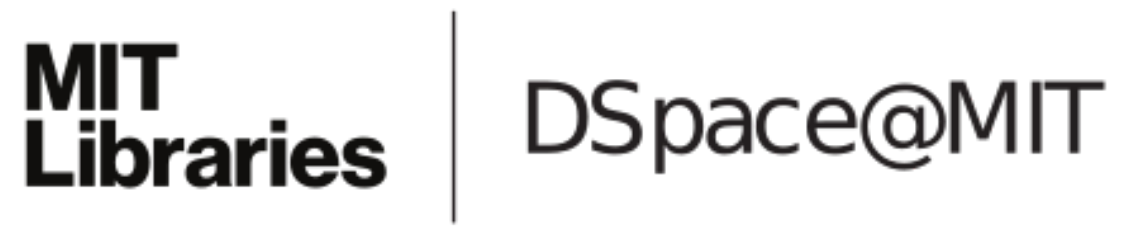

\author{
MIT Open Access Articles
}

On a bilinear Strichartz estimate on irrational tori

The MIT Faculty has made this article openly available. Please share how this access benefits you. Your story matters.

Citation: Fan, Chenjie et al. "On a Bilinear Strichartz Estimate on Irrational Tori." Analysis \& PDE 11, 4 (January 2018): 919-944 @ 2018 Mathematical Sciences Publishers

As Published: http://dx.doi.org/10.2140/apde.2018.11.919

Publisher: Mathematical Sciences Publishers

Persistent URL: http://hdl.handle.net/1721.1/115979

Version: Author's final manuscript: final author's manuscript post peer review, without publisher's formatting or copy editing

Terms of use: Creative Commons Attribution-Noncommercial-Share Alike 


\title{
ON A BILINEAR STRICHARTZ ESTIMATE ON IRRATIONAL TORI
}

\author{
CHENJIE FAN F $^{*}$, GIGLIOLA STAFFILANI ${ }^{\dagger} *$, HONG WANG ${ }^{\S}$, AND BOBBY WILSON $\sharp$
}

\begin{abstract}
We prove a bilinear Strichartz type estimate for irrational tori via a decoupling type argument, 4, recovering and generalizing the result of [7. As a corollary, we derive a global well-posedness result for the cubic defocusing NLS on two dimensional irrational tori with data of infinite energy.
\end{abstract}

\section{INTRODUCTION}

In [4] Bourgain and Demeter proved the full range of Strichartz estimates for the Schrödingier equation on tori as a consequence of the $L^{2}$ decoupling theorem. In this paper we prove in full generality the analog of the improved Strichartz estimate that first appeared in [7] for rational tori.

1.1. Statement of the problem and main results. Let $\mathbb{T}=\mathbb{R} / \mathbb{Z}$ be the one dimensional torus, and let $\alpha_{1}, . ., \alpha_{d-1} \in[1 / 2,1]$, we define $d$-dimensional torus $\mathbb{T}^{d}$ as $\mathbb{T}^{d}=\mathbb{T} \times \alpha_{1} \mathbb{T} \times \cdots \times \alpha_{d-1} \mathbb{T}$. We say that the torus is irrational if at least on $\alpha_{i}$ is irrational. The torus is rational otherwise. For any $\lambda \geq 1$, we define $\mathbb{T}_{\lambda}^{d}$ as a rescaling of $\mathbb{T}^{d}$ by $\lambda$, i.e. $\mathbb{T}_{\lambda}^{d}=\lambda \mathbb{T}^{d}=(\lambda \mathbb{T}) \times\left(\alpha_{1} \lambda \mathbb{T}\right) \ldots \times\left(\alpha_{d-1} \lambda \mathbb{T}\right)$.

When $\lambda \rightarrow \infty$, one should think $\mathbb{T}_{\lambda}$ as a large torus approximating $\mathbb{R}^{d}$. We consider the following linear Schrödinger equation on $\mathbb{T}_{\lambda}$, We consider the following Cauchy problem for the linear Schrödinger equation on $\mathbb{T}_{\lambda}^{d}$,

$$
\left\{\begin{array}{l}
i u_{t}-\Delta u=0,(t, x) \in \mathbb{R} \times \mathbb{T}_{\lambda}^{d} \\
u(0, x)=u_{0}, u_{0} \in L^{2}\left(\mathbb{T}_{\lambda}^{d}\right) .
\end{array}\right.
$$

Let $U_{\lambda}(t) u_{0}$ be the solution to (1.1), and let $\Lambda_{\lambda}:=\frac{1}{\lambda}\left(\mathbb{Z} \times \frac{1}{\alpha_{1}} \mathbb{Z} \times \cdots \times \frac{1}{\alpha_{d-1}} \mathbb{Z}\right)$. One has

$$
U_{\lambda}(t) u_{0}(x)=\frac{1}{\lambda^{d / 2}} \sum_{k \in \Lambda_{\lambda}} e^{2 \pi k i x-|2 \pi k|^{2} i t} \widehat{u}_{0}(k) .
$$

Our main theorem is the following bi-linear refined Strichartz estimate.

Theorem 1.1. Let $\phi_{1}, \phi_{2} \in L^{2}\left(\mathbb{T}_{\lambda}\right)$ be two initial data such that supp $\hat{\phi}_{i} \subset\left\{k:|k| \sim N_{i}\right\}, i=1,2$, for some large $N_{1} \geq N_{2}$, and let $\eta(t)$ be a time cut-off function, supp $\eta \subset[0,1]$. Then

when $d=2$,

$$
\left\|\eta(t) U_{\lambda} \phi_{1} \cdot \eta(t) U_{\lambda} \phi_{2}\right\|_{L_{x, t}^{2}} \lesssim N_{2}^{\epsilon}\left(\frac{1}{\lambda}+\frac{N_{2}}{N_{1}}\right)^{1 / 2}\left\|\phi_{1}\right\|_{L^{2}}\left\|\phi_{2}\right\|_{L^{2}}
$$

when $d \geq 3$

$$
\left\|\eta(t) U_{\lambda} \phi_{1} \cdot \eta(t) U_{\lambda} \phi_{2}\right\|_{L_{x, t}^{2}} \lesssim N_{2}^{\epsilon}\left(\frac{N_{2}^{d-3}}{\lambda}+\frac{N_{2}^{d-1}}{N_{1}}\right)^{1 / 2}\left\|\phi_{1}\right\|_{L^{2}}\left\|\phi_{2}\right\|_{L^{2}}
$$

We note that when $d=2, N_{1}=N_{2}, \lambda=1$, estimate (1.3) recovers the Strichartz inequality for the (irrational) torus after an application of Hölder's inequality, up to an $N_{2}^{\epsilon}$-loss. When $\lambda \rightarrow \infty$, estimate (1.3), (1.4) consistent with the billinear Strichartz inequality in $\mathbb{R}^{d+1}$, 2]. Up to the $N_{2}^{\epsilon}$-loss, inequality (1.3) is sharp.

Furthermore, when $\lambda \geq N_{1}$, the estimates fall into the so-called semiclassical regime in which the geometry of $\mathbb{T}_{\lambda}$ is irrelevant. We refer to the work of Hani, [1], for same estimate (without $N_{2}^{\epsilon}$ loss) on general compact manifolds.

\footnotetext{
* Chenjie Fan and Gigliola Staffilani are partially supported by NSF DMS 1362509 and DMS 1462401.

${ }^{\ddagger}$ Department of Mathematics, University of Chicago, 5734 S University Ave, Chicago, IL 60637, USA. email: cjfan@math.uchicago.edu. $\dagger$ Department of Mathematics, Massachusetts Institute of Technology, 77 Massachusetts Ave, Cambridge, MA 02139-4307 USA. email: gigliola@math.mit.edu.

$\S$ Department of Mathematics, Massachusetts Institute of Technology, 77 Massachusetts Ave, Cambridge, MA 02139-4307 USA. email: hongwang@mit.edu.

\#Department of Mathematics, Massachusetts Institute of Technology, 77 Massachusetts Ave, Cambridge, MA 02139-4307 USA. email: blwilson@mit.edu.
} 
On the torus, our results improves the estimate in [11] for $\lambda \leq N_{1}$. Estimate (1.3), (1.4) rely on the geometry of torus and cannot hold on general compact manifolds.

Remark 1.2. It may also be interesting to consider trilinear estimates. In fact when one considers the quintic nonlinear Schrödinger equation as in [12] and [13], trilinear estimates are fundamental. See also [15].

We will derive Theorem 1.1 from some bilinear decoupling type estimates. We first introduce some basic notations.

Let $P$ be the truncated paraboloid in $\mathbb{R}^{d+1}$,

$$
P=\left\{\left(\xi,|\xi|^{2}\right): \xi \in \mathbb{R}^{d},|\xi| \lesssim 1\right\} .
$$

For any function $f$ supported on $P$, we define

$$
E f=\widehat{f d \sigma}
$$

where $\sigma$ is the measure on $P$.

Note a function supported on $P$ can be naturally understood as a function supported on the ball $B=\{\xi \in$ $\left.\mathbb{R}^{d},|\xi| \lesssim 1\right\}$.

By a slight abuse of notation, for a function $f$ supported in the ball $B$ in $\mathbb{R}^{d}$, we also define

$$
E f(x, t)=\int_{B} e^{-2 \pi i\left(\xi \cdot x+|\xi|^{2} t\right)} f(\xi) d \xi .
$$

One can see that the two definitions of $E f$ are essentially the same since $P$ projects onto $B$.

We decompose $P$ as a finitely overlapping union of caps $\theta$ of radius $\delta$. Here a cap $\theta$ of radius $\delta$ is the set $\theta=\left\{\xi \in P,\left|\xi-\xi_{0}\right| \lesssim \delta\right\}$ for some fixed $\xi_{0} \in P$. We define $E f_{\theta}=\widehat{f_{\theta} d \sigma}$, where $f_{\theta}$ is $f$ restricted to $\theta$. We use a similar definition also when $f$ is a function supported on the unit ball in $\mathbb{R}^{d}$. We have $E f=\sum_{\theta} E f_{\theta}$.

Now, we are ready to state our main decoupling type estimate.

Theorem 1.3. Given $\lambda \geq 1, N_{1} \geq N_{2} \geq 1$. Let $f_{1}$ be supported on $P$ where $|\xi| \sim 1$, and let $f_{2}$ be supported on $P$ where $|\xi| \sim \frac{N_{2}}{N_{1}}$. Let $\Omega=\left\{(t, x) \in\left[0, N_{1}^{2}\right] \times\left[0,\left(\lambda N_{1}\right)^{2}\right]^{d}\right\}$. For a finitely overlapping covering of the ball $B=\{|\xi| \leq 1\}$ of caps $\{\theta\},|\theta|=\frac{1}{\lambda N_{1}}$, we have the following estimate. For any small $\epsilon>0$, when $d=2$,

$$
\left\|E f_{1} E f_{2}\right\|_{L_{\text {avg }}^{2}\left(w_{\Omega}\right)} \lesssim_{\epsilon}\left(N_{2}\right)^{\epsilon} \lambda^{d / 2}\left(\frac{1}{\lambda}+\frac{N_{2}^{d-1}}{N_{1}}\right)^{1 / 2} \prod_{j=1}^{2}\left(\sum_{|\theta|=\frac{1}{\lambda N_{1}}}\left\|E f_{j, \theta}\right\|_{L_{\text {avg }}^{4}\left(w_{\Omega}\right)}^{2}\right)^{1 / 2}
$$

when $d \geq 3$,

$$
\left\|E f_{1} E f_{2}\right\|_{L_{\text {avg }}^{2}\left(w_{\Omega}\right)} \lesssim_{\epsilon}\left(N_{2}\right)^{\epsilon} \lambda^{d / 2}\left(\frac{N_{2}^{d-3}}{\lambda}+\frac{N_{2}^{d-1}}{N_{1}}\right)^{1 / 2} \prod_{j=1}^{2}\left(\sum_{|\theta|=\frac{1}{\lambda N_{1}}}\left\|E f_{j, \theta}\right\|_{L_{\text {avg }}^{4}\left(w_{\Omega}\right)}^{2}\right)^{1 / 2},
$$

where $w_{\Omega}$ is a weight adapted to $\Omega$.

The presence of weight $w$ in these estimates is standard. We list the basic property of $w$ in Section [1.5, and one can refer to [5] for more details. The notation $L_{\text {avg }}\left(w_{\Omega}\right)^{2}$ is explained in notation subsection below, subsection 1.4.

The proof of Theorem 1.3 gives another proof of the linear decoupling theorem in [4] in dimension $d=2$, and does not rely on multilinear-Kakeya or multilinear restriction theorems in $\mathbb{R}^{3}$. The proof of Theorem 1.3 in dimension $d \geq 3$ relies instead on linear decoupling in $\mathbb{R}^{d+1},[4$.

Remark 1.4. The estimate in Theorem 1.1, Theorem 1.3 is sharp up to an $N_{2}^{\epsilon}$. See Section $A$ for examples.

Remark 1.5. The $N_{2}^{\epsilon}$ loss in Theorem 1.1 is typical if one wants to directly use a decoupling type argument. It may be possible to remove $N_{2}^{\epsilon}$ in the mass supercritical setting, (in our case, this means $d \geq 3$ ), using the approach in [14, where the scale invariant Strichartz estimates are studied.

Remark 1.6. Similar bilinear estimates for dimension $d \geq 3$ were also considered in [14] for non-rescaled tori, see Lemma 3.3. On the other hand in this work we also consider the $d=2$ case which is mass critical.

1.2. Acknowledgment. We thank Larry Guth for very helpful discussions during the course of this work. 
1.3. Background and motivation. The system (1.1) and the bilinear estimates (1.3) and (1.4) naturally appear in the study of the following nonlinear Schrödinger equation on the non-rescaled tori:

$$
\left\{\begin{array}{l}
i u_{t}+\Delta u=|u|^{2} u \\
u(0)=u_{0} \in H^{s}\left(\mathbb{T}^{d}\right) .
\end{array}\right.
$$

Let us focus for a moment on the $d=2$ case. The Cauchy problem is said to be locally well-posed in $H^{s}\left(\mathbb{T}^{d}\right)$ if for any initial data $u_{0} \in H^{s}\left(\mathbb{T}^{d}\right)$ there exists a time $T=T\left(\left\|u_{0}\right\|_{s}\right)$ such that a unique solution to the initial value problem exists on the time interval $[0, T]$. We also require that the data to solution map is continuous from $H^{s}\left(\mathbb{T}^{d}\right)$ to $C_{t}^{0} H_{x}^{s}\left([0, T] \times \mathbb{T}^{d}\right)$. If $T=\infty$, we say that a Cauchy problem is globally well-posed.

The initial value problem (1.10) is locally well-posed for initial data $u_{0} \in H^{s}, s>0$ via Strichartz estimates. Note that using iteration, by the energy conservation law, i.e.

$$
E(u(t))=E\left(u_{0}\right)=\frac{1}{2} \int|\nabla u|^{2}+\frac{1}{4} \int|u|^{4},
$$

all initial data in $H^{1}\left(\mathbb{T}^{2}\right)$ give rise to a global solution. Next, by the nowadays standard I-method, [6], by considering a modified version of the energy, in the rational torus case, it was proved in [7] that (1.10) is indeed globally wellposedness for initial data in $H^{s}, s>2 / 3$. The key estimate there was in fact (1.3) for linear solutions on rescaled tori, which we prove here to be available also for irrational tori.

The proof for (1.3) presented in [7] is only for rational tori since it relies on certain types of counting lemmata that cannot directly work on irrational tori. One of the main purpose of this work in fact is to extend results on rational tori to irrational ones.

Based on the discussion we just made, as a corollary of Theorem 1.1, we have

Corollary 1.7. The initial value problem (1.10) defined on any torus $\mathbb{T}^{2}$ is globally well-posed for initial data in $H^{s}\left(\mathbb{T}^{2}\right)$ with $s>2 / 3$.

Remark 1.8. Results such as Corollary [1.7 usually also give a control on the growth of Sobolev norms of the global solutions. We do not address this particular question here. We instead refer the reader to the recent work [8].

The original Strichartz estimates needed to prove the local well-posedness of Cauchy problems such as (1.10) were first obtained in [1] via number theoretical related counting arguments for rational tori. Recently, the striking proof of the $L^{2}$ decoupling Theorem, [4, provided a completely different approach from which all the desired Strichartz estimates on tori, both rational and irrational, follow. This approach in particular does not depend on counting lattice points. See also the work [10] and [9]. The method of proof we implement in this present work is mostly inspired by [4] and the techniques used to prove the $L^{2}$ decoupling Theorem.

We quickly recall the main result in $\left[4\right.$. Let $P$ be a unit parabola in $\mathbb{R}^{d+1}$, covered by finitely overlapping caps $\theta$ of radius $\frac{1}{R}$. Let $f$ be a function defined on $P$, then one has for any $\epsilon>0$ small,

$$
\|E f\|_{L^{p}\left(w_{B^{2}}\right)} \lesssim \epsilon R^{\epsilon}\left(R^{2}\right)^{d / 4-\frac{d+2}{2 p}}\left(\sum_{\theta}\left\|E f_{\theta}\right\|_{L^{p}\left(B_{R^{2}}\right)}^{2}\right)^{1 / 2}, \quad p \geq \frac{2(d+2)}{d} .
$$

Note that (1.11) corresponds to Theorem 1.1 in [4, and the dimension $n$ in the estimate (2) there corresponds to our $d+1$. Also note that the linear decoupling (1.11) not only works for those $f$ exactly supported on $P$, but those $f$ supported in a $R^{-2}$ neighborhood of $P$, and in this case, cap $\theta$ would be replaced by the $R^{-2}$ neighborhood of the original $\theta$, see Theorem 1.1 in [4].

We remark that one key feature of this decoupling type estimate is that one needs to work on a larger scale in physical space, i.e. the scale $R^{2}$ rather than $R$, in order to observe the decoupling phenomena. The proper observational scale dictated by Heisenberg's uncertainty principle is $R$.

Indeed, one principle, which is usually called parallel decoupling, indicates that if decoupling happens in a small region, then decoupling happens in a large region as well. We state a bilinear version the parallel decoupling below.

Lemma 1.9 (4, [5]). Let $D$ be a domain, and $D=D_{1} \cup D_{2} \ldots \cup D_{J}, D_{i} \cap D_{j}=\emptyset$. If for some constant $A>0$ and for function $h_{1}, h_{2}$, defined on the unit parabola, one has

$$
\left\|E h_{1} E h_{2}\right\|_{L_{a v g}^{2}\left(w_{D_{i}}\right)} \leq A \prod_{j=1}^{2}\left(\sum_{|\theta|=\frac{1}{\lambda N_{1}}}\left\|E h_{j, \theta}\right\|_{L_{a v g}^{4}\left(w_{D_{i}}\right)}^{2}\right)^{1 / 2} i=1, \ldots, J
$$


then one also has

$$
\left\|E h_{1} E h_{2}\right\|_{L_{a v g}^{2}\left(w_{D}\right)} \leq A \prod_{j=1}^{2}\left(\sum_{|\theta|=\frac{1}{\lambda N_{1}}}\left\|E h_{j, \theta}\right\|_{L_{a v g}^{4}\left(w_{D}\right)}^{2}\right)^{1 / 2} .
$$

The proof of this particular formulation of parallel decoupling follows by Minkowski's inequality.

As it exists, parallel decoupling is a principle rather than a concrete lemma. We state the version here solely for concreteness. It should be easy to generalize the lemma under different conditions.

1.4. Notation. We write $A \lesssim B$ if $A \leq C B$, for a constant $C>0, A \sim B$ if both $A \lesssim B$ and $B \lesssim A$. We say $A \lesssim_{\epsilon} B$ if the constant $C$ depends on $\epsilon$. Similarly for $A \sim_{\epsilon} B$. For a Borel set, $E \subset \mathbb{R}^{d}$, we denote that diameter of $E$ by $|E|$ and the Lebesgue measure of $E$ by $m(E)$.

We will use the usual function space $L^{p}$. We also use a (weighted) average version of $L^{p}$ space, i.e

$$
\|g\|_{L_{a v g}^{p}(A)}=\left(f_{A}|g|^{p}\right)^{1 / p}:=\left(\frac{1}{m(A)} \int_{A}|g|^{p}\right)^{1 / p}
$$

and

$$
\|g\|_{L_{a v g}^{p}\left(w_{A}\right)}=\left(\frac{1}{m(A)} \int|g|^{p} w_{A}\right)^{1 / p},
$$

where $w_{A}$ is a weight function described below.

For any function $f$, we use $\hat{f}$ to denote its Fourier transform. When we say unit ball, we refer to a ball of radius $r \sim 1$. We will often identify a torus as a bounded domain in Euclidean space, for example, we will view $(\mathbb{R} / \mathbb{Z})^{d}$ as $[0,1]^{d} \subset \mathbb{R}^{d}$. In this work, $\Omega$ is used to denote the domain $\left[0, N_{1}^{2}\right] \times\left[0,\left(\lambda N_{1}\right)^{2}\right]^{d} \subset \mathbb{R}^{d+1}$.

1.5. The weight $w_{A}$. If $h$ is a Schwartz function whose Fourier transform, $\hat{h}$, is supported in a ball of radius $1 / R$, we expect $h$ be essentially constant on balls of radius $R$, and morally

$$
\|h\|_{L_{a v g}^{p}\left(B_{R}\right)} \sim\|h\|_{L_{a v g}^{2}\left(B_{R}\right)} \sim\|h\|_{L^{\infty}\left(B_{R}\right)} .
$$

Expression (1.14) is not rigorous, and the introduction of the weight $w_{B_{R}}$ is a standard way to overcome this technical difficulty. We refer to Lemma 4.1 in [5] for more detailed discussion of the weight function.

For any bounded open convex set $A$, the weight function $w_{A}$, might change from line to line, from the left hand side of the inequality to the right hand side, satisfies the same properties:

- $\int w_{A} \sim m(A)$.

- $w_{A} \gtrsim 1$ on $A$, and rapidly (polynomial type) decay outside $A$.

We will usually define $A$ to be a ball, or the product of balls in this paper.

Furthermore, let $B_{R}$ be a ball centered at 0 , and let $\mu_{B_{R}}$ be a function such that $\widehat{\mu_{B_{R}}}$ is about $\frac{1}{m\left(B_{1 / R}\right)}$ on $B_{1 / R}$, and supported in $B_{2 / R}$, then $\mu_{B_{R}}$ is about 1 on $B_{R}$, decays faster than any polynomial outside of $B_{R}$. $\mu_{B_{R}}^{2}$ is positive, decays faster than any polynomial outside of $B_{R}$ and fourier supported in $B_{4 / R}$, We take translations $B^{\prime}$ of $B_{R}$ to cover the whole space, we note $\mu_{B^{\prime}}$ as the corresponding translation of $\mu_{B_{R}}$ and $w_{B_{R}}\left(B^{\prime}\right)=\max _{x \in B^{\prime}} w_{B_{R}}$, we have the following useful property,

$$
w_{B_{R}}(x) \leq \sum_{B^{\prime}} w_{B_{R}}\left(B^{\prime}\right) 1_{B^{\prime}}(x) \lesssim \sum_{B^{\prime}} w_{B_{R}}\left(B^{\prime}\right) \mu_{B^{\prime}}^{2}(x) \lesssim w_{B_{R}}(x)
$$

The last inequality follows from the fact that $\mu_{B^{\prime}}^{2}$ decays faster than any polynomial outside of $B^{\prime}$.

Lemma 1.10. For a function $f$ supported in $B_{1 / R}$, for any $p<\infty$,

$$
\|E f\|_{L^{\infty}\left(B_{R}\right)} \lesssim\|E f\|_{L_{a v g}^{p}\left(\mu_{B_{R}}\right)} .
$$

We refer the proof to Corollary 4.3 in [5] with the weight on the left hand side being $1_{B_{R}}$ so that on the right hand side we have a fast decay weight.

Remark 1.11. In general, Lemma 1.10 should hold for any convex set $A$ and the dual convex body $A^{*}$. 


\section{Proof of Theorem 1.1 assuming Theorem 1.3}

Assume Theorem 1.3, let us prove Theorem 1.1. The argument below comes from the proof of discrete restriction and Strichartz estimate on irrational tori assuming the $L^{2}$ decoupling estimate, see Theorem 2.2, Theorem 2.3 in 4]. The argument originally comes as observation due to Bourgain [3]. We record it here for completeness.

Let $\phi_{1}, \phi_{2}$ be as in Theorem 1.1. We rescale $\phi_{1}$ to be supported in the unit ball and rescale $\phi_{2}$ to be supported in a ball of radius $\sim \frac{N_{2}}{N_{1}}$. Recall,

$$
U_{\lambda}(t) \phi_{j}(x, t)=\frac{1}{\lambda^{d / 2}} \sum_{k \in \Lambda_{\lambda}, k \sim N_{1}} e^{2 \pi i k \cdot x-|2 \pi k|^{2} t} \widehat{\phi}_{j}(k) .
$$

We perform a change of variables $\xi=\frac{k}{N_{1}}$ and we let

$$
h_{j}(\tau)=\frac{1}{\lambda^{d / 2}} \sum_{\xi \in \Lambda_{\lambda N_{1}},|\xi| \sim 1} \widehat{\phi}_{j}\left(\xi N_{1}\right) \delta_{\xi}(\tau), \quad j=1,2 .
$$

Note one can directly check that

$$
U_{\lambda}(t) \phi_{j}(x, t)=E h_{j}\left(-2 \pi N_{1} x,(2 \pi)^{2} N_{1}^{2} t\right) .
$$

Without loss of generality, we suppress the constants $-2 \pi$ and $(2 \pi)^{2}$.

Let $Q_{0}=\left[0, N_{1}^{2}\right] \times \mathbb{T}_{\lambda N_{1}}^{d}$ and let us view $\mathbb{T}_{\lambda N_{1}}^{d}$ as a compact set in $\mathbb{R}^{d}$. In particular, one can construct the associated weight function $w_{Q_{0}}$. Direct computation (via change of variables) gives

$$
\left.\| U_{\lambda}(t) \phi_{1}\right) U_{\lambda}(t) \phi_{2}\left\|_{L^{2}\left([0,1] \times \mathbb{T}_{\lambda}^{d}\right)} \sim N_{1}^{-\frac{d+2}{2}} m\left(Q_{0}\right)^{1 / 2}\right\| E h_{1} E h_{2} \|_{L_{a v g}^{2}\left(Q_{0}\right)}
$$

and due to the the periodicity of $E h_{i}, i=1,2$, one has

$$
\left\|E h_{1} E h_{2}\right\|_{L_{\text {avg }}^{2}(\Omega)}=\left\|E h_{1} E h_{2}\right\|_{L_{\text {avg }}^{2}\left(Q_{0}\right)} .
$$

For a covering $\{\theta\}$ of caps of radius $\frac{1}{\lambda N_{1}}$, each cap $\theta$ contains at most one $\xi_{\theta} \in \Lambda_{\lambda N_{1}}$, corresponding to $k_{\theta}=$ $N_{1} \xi_{\theta} \in \Lambda_{\lambda}$, then

$$
\left\|E h_{j, \theta}\right\|_{L_{a v g}^{4}\left(w_{Q_{0}}\right)} \sim h_{j}\left(\xi_{\theta}\right) \sim \frac{1}{\lambda^{d}} \widehat{\phi}_{j}\left(k_{\theta}\right)
$$

and

$$
\begin{aligned}
\prod_{j=1}^{2}\left(\sum_{|\theta|=\frac{1}{\lambda N_{1}}}\left\|E h_{j}\right\|_{\left.L_{a v g}^{4}\left(w_{Q_{0}}\right)\right)^{1 / 2}}^{2}\right. & \sim \lambda^{-d} \prod_{j=1}^{2}\left(\frac{1}{\lambda^{d}} \sum_{k \in \Lambda_{\lambda}}\left|\widehat{\phi}_{j}(k)\right|^{2}\right)^{1 / 2} \\
& \sim \lambda^{-d}\left\|\phi_{1}\right\|_{L^{2}}\left\|\phi_{2}\right\|_{L^{2}} .
\end{aligned}
$$

For convenience of notation let

$$
D_{\lambda, N_{1}, N_{2}}:=\left\{\begin{array}{l}
\frac{1}{\lambda}+\frac{N_{2}}{N_{1}}, \text { when } d=2, \\
\frac{N_{2}^{d-3}}{\lambda}+\frac{N_{2}^{d-1}}{N_{1}}, \text { when } d \geq 3
\end{array}\right.
$$

Recall that $\Omega=\left[0, N_{1}\right]^{2} \times\left[0,\left(\lambda N_{1}\right)^{2}\right]^{d}$, we apply Theorem 1.3 with $f_{j}=h_{j}$, and we have

$$
\left\|E h_{1} E h_{2}\right\|_{L_{a v g}^{2}\left(w_{\Omega}\right)} \lesssim_{\epsilon}\left(N_{2}\right)^{\epsilon} \lambda^{d / 2} D_{\lambda, N_{1}, N_{2}}^{1 / 2} \prod_{j=1}^{2}\left(\sum_{|\theta|=\frac{1}{\lambda N_{1}}}\left\|E h_{j, \theta}\right\|_{L_{\text {avg }}^{4}\left(w_{\Omega}\right)}^{2}\right)^{1 / 2} .
$$

Note that $\Omega$ can be covered by $Q$ such that $\{Q\}$ are finitely overlapping and each $Q$ is a translation of $Q_{0}$. Since $E h_{j}$ are periodic on $x$, estimate (2.7) is equivalent to

$$
\left\|E h_{1} E h_{2}\right\|_{L_{a v g}^{2}\left(w_{Q_{0}}\right) \lesssim \epsilon}\left(N_{2}\right)^{\epsilon} \lambda^{d / 2} D_{\lambda, N_{1}, N_{2}}^{1 / 2} \prod_{j=1}^{2}\left(\sum_{|\theta|=\frac{1}{\lambda N_{1}}}\left\|E h_{j, \theta}\right\|_{L^{4}\left(w_{Q_{0}}\right)}^{2}\right)^{1 / 2} .
$$


Plugging (2.8) into (2.4) gives

$$
\begin{aligned}
& \left\|U_{\lambda}(t) \phi_{1} U_{\lambda}(t) \phi_{2}\right\|_{L^{2}\left([0,1] \times \mathbb{T}_{\lambda}^{d}\right)} \\
& \lesssim N_{1}^{-\frac{d+2}{2}} \cdot N_{1} m\left(\mathbb{T}_{\lambda N_{1}}^{d}\right)^{1 / 2} \lambda^{-d} \cdot\left(N_{2}\right)^{\epsilon} \lambda^{d / 2} D_{\lambda, N_{1}, N_{2}}^{1 / 2}\left\|\phi_{1}\right\|_{L^{2}}\left\|\phi_{2}\right\|_{L^{2}} \\
& \sim\left(N_{2}\right)^{\epsilon} D_{\lambda, N_{1}, N_{2}}^{1 / 2}\left\|\phi_{1}\right\|_{L^{2}}\left\|\phi_{2}\right\|_{L^{2}}
\end{aligned}
$$

and Theorem 1.1 follows.

The rest of the paper details the proof of Theorem 1.3

\section{An overview of the proof of Theorem 1.3}

First, we reduce the proof of Theorem 1.3 to the following proposition.

Proposition 3.1. Let $\tau_{1}$ be a cap of radius $\frac{N_{2}}{N_{1}}$ supported at $\xi$ and $|\xi| \sim 1$. Let $\tau_{2}$ be a cap of radius $\frac{N_{2}}{N_{1}}$ supported at $\xi$ with $|\xi| \sim \frac{N_{2}}{N_{1}}$. Let $f_{j}$ be a function supported in $\tau_{j}$, then for any small $\epsilon>0$,

when $d=2$

$$
\left\|E f_{1} E f_{2}\right\|_{L_{a v g}^{2}\left(w_{\Omega}\right)} \lesssim_{\epsilon}\left(N_{2}\right)^{\epsilon} \lambda^{d / 2}\left(\frac{1}{\lambda}+\frac{N_{2}}{N_{1}}\right)^{1 / 2} \prod_{j=1}^{2}\left(\sum_{|\theta|=\frac{1}{\lambda N_{1}}, \theta \subset \tau_{j}}\left\|E f_{j, \theta}\right\|_{L_{\text {avg }}^{4}\left(w_{\Omega}\right)}^{2}\right)^{1 / 2}
$$

when $d \geq 3$,

$$
\left\|E f_{1} E f_{2}\right\|_{L_{a v g}^{2}\left(w_{\Omega}\right)} \lesssim_{\epsilon}\left(N_{2}\right)^{\epsilon} \lambda^{d / 2}\left(\frac{N_{2}^{d-3}}{\lambda}+\frac{N_{2}^{d-1}}{N_{1}}\right)^{1 / 2} \prod_{j=1}^{2}\left(\sum_{|\theta|=\frac{1}{\lambda N_{1}}, \theta \subset \tau_{j}}\left\|E f_{j, \theta}\right\|_{L_{\text {avg }}^{4}\left(w_{\Omega}\right)}^{2}\right)^{1 / 2}
$$

Now, let $f_{1}, f_{2}$ be as in Proposition 3.1. We define $K_{0}\left(\lambda, N_{1}, N_{2}\right)$ to be the best constant such that

$$
\left\|E f_{1} E f_{2}\right\|_{L_{a v g}^{2}\left(w_{\Omega}\right)} \leq \lambda^{d / 2} K_{0}\left(\lambda, N_{1}, N_{2}\right) \prod_{j=1}^{2}\left(\sum_{|\theta|=\frac{1}{\lambda N_{1}}}\left\|E f_{j, \theta}\right\|_{L_{a v g}^{4}\left(w_{\Omega}\right)}^{2}\right)^{1 / 2} .
$$

We also let $\tilde{K}\left(\lambda, N_{1}, N_{2}\right)$ and $K\left(\lambda, N_{1}, N_{2}\right)$ be defined as the best constants such that

$$
\begin{gathered}
\left\|E f_{1} E f_{2}\right\|_{L_{\text {avg }}^{2}\left(w_{\left[0, N_{1}^{2}\right] \times\left[0, \lambda N_{1}\right]^{d}}\right)} \leq \lambda^{d / 2} \tilde{K}\left(\lambda, N_{1}, N_{2}\right) \prod_{j=1}^{2}\left(\sum_{|\theta|=\frac{1}{\lambda N_{1}}}\left\|E f_{j, \theta}\right\|_{L_{a v g}^{4}\left(w_{\left[0, N_{1}^{2}\right] \times\left[0, \lambda N_{1}\right]^{d}}\right)}^{2}\right)^{1 / 2}, \\
\left\|E f_{1} E f_{2}\right\|_{L_{a v g}^{2}\left(w_{N_{N}}\right)} \leq \lambda^{d / 2} K\left(\lambda, N_{1}, N_{2}\right) \prod_{j=1}^{2}\left(\sum_{|\theta|=\frac{1}{\lambda N_{1}}}\left\|E f_{j, \theta}\right\|_{L_{a v g}^{4}\left(w_{B_{N_{1}^{2}}}\right)}^{2}\right)^{1 / 2} .
\end{gathered}
$$

Below we will prove that

$$
\begin{aligned}
& K_{0}\left(\lambda, N_{1}, N_{2}\right) \lesssim N_{2}^{\epsilon}\left(\frac{1}{\lambda}+\frac{N_{2}}{N_{1}}\right)^{1 / 2}, \quad d=2, \\
& K_{0}\left(\lambda, N_{1}, N_{2}\right) \lesssim N_{2}^{\epsilon}\left(\frac{N_{2}^{d-3}}{\lambda}+\frac{N_{2}^{d-1}}{N_{1}}\right)^{1 / 2}, \quad d \geq 3 .
\end{aligned}
$$

We point out here that by parallel decoupling and Lemma 1.9 one always has

$$
K_{0}\left(\lambda, N_{1}, N_{2}\right) \lesssim K\left(\lambda, N_{1}, N_{2}\right), \quad K_{0}\left(\lambda, N_{1}, N_{2}\right) \lesssim \tilde{K}\left(\lambda, N_{1}, N_{2}\right) .
$$

The proof of Proposition 3.1 or equivalently (3.6) proceeds as follows. We first show

Lemma 3.2. When $\lambda \geq N_{1}$,

$$
\tilde{K}\left(\lambda, N_{1}, N_{2}\right) \lesssim N_{2}^{\epsilon} \frac{N_{2}^{(d-1) / 2}}{N_{1}^{1 / 2}} .
$$

Note that when $\lambda \geq N_{1}$, Proposition 3.1 follows from (3.7) and Lemma 3.2 .

Then, we show 
Lemma 3.3. When $\lambda \leq N_{1}$,

$$
\begin{aligned}
& K\left(\lambda, N_{1}, N_{2}\right) \lesssim N_{2}^{\epsilon}\left(\frac{1}{\lambda}+\frac{N_{2}}{N_{1}}\right)^{1 / 2}, \quad d=2, \\
& K\left(\lambda, N_{1}, N_{2}\right) \lesssim N_{2}^{\epsilon}\left(\frac{N_{2}^{d-3}}{\lambda}+\frac{N_{2}^{d-1}}{N_{1}}\right)^{1 / 2}, \quad d=3 .
\end{aligned}
$$

From (3.7), clearly Proposition 3.1 follows from Lemma 3.2 and Lemma 3.3 ,

The proof of Lemma 3.3 in dimension $d=2$ relies on induction (of scale $N_{2}$ ). The proof of Lemma 3.3 in dimension in $d \geq 3$ is easier and more straightforward, (in some sense, it also relies on induction, but it is enough to induct only once.)

We first show the base case:

Lemma 3.4. When $\lambda \leq N_{1}$ and $N_{2} \lesssim 1, K\left(\lambda, N_{1}, N_{2}\right) \lesssim \frac{1}{\lambda^{1 / 2}}$.

Lemma 3.4 is not as useful in dimension $d \geq 3$, we indeed have a better estimate:

Lemma 3.5. When $d \geq 3, \lambda \leq N_{1}$ and $\lambda \leq \frac{N_{1}}{N_{2}^{2}}, K\left(\lambda, N_{1}, N_{2}\right) \lesssim\left(\frac{N_{2}^{d-3}}{\lambda}\right)^{1 / 2}$.

We then show the following lemma, which ensures that we only need to induct until $\lambda \leq \frac{N_{1}}{N_{2}}$, when $d=2$, and until $\frac{N_{1}}{N_{2}}$ when $d \geq 3$.

Lemma 3.6. Let $\lambda \leq N_{1}$.

Let $d=2$. Assume we have that $K\left(\lambda, N_{1}, N_{2}\right) \leq \lambda^{-1 / 2}$ when $\lambda<\frac{N_{1}}{N_{2}}$. Then

$$
K\left(\lambda, N_{1}, N_{2}\right) \leq N_{2}^{\epsilon} \frac{N_{2}^{\frac{d-1}{2}}}{N_{1}^{1 / 2}} \quad \text { when } \quad \lambda \geq \frac{N_{1}}{N_{2}} .
$$

Let $d \geq 3$. Assume we have that $K\left(\lambda, N_{1}, N_{2}\right) \leq\left(\frac{N_{2}^{d-3}}{\lambda}\right)^{1 / 2}$ when $\lambda<\frac{N_{1}}{N_{2}^{2}}$. Then

$$
K\left(\lambda, N_{1}, N_{2}\right) \leq N_{2}^{\epsilon} \frac{N_{2}^{\frac{d-1}{2}}}{N_{1}^{1 / 2}} \quad \text { when } \quad \lambda \geq \frac{N_{1}}{N_{2}^{2}} .
$$

Note that when $d \geq 3$, Lemma 3.5 and Lemma 3.6 imply Lemma 3.3. In dimension $d=2$, we use induction (we rely on the so-called parabolic rescaling) to finish the proof of Lemma 3.3 .

We end this section with an outline of the structure of the rest of the paper. We show that Proposition 3.1 implies Theorem 1.3 in Section 4. Lemma 3.2. Lemma 3.4 Lemma 3.6 all rely on the exploration of the so-called transversality which essentially allow us to reduce the dimensionality of the problem. We first explore transversality in Section 5 and then we prove Lemma 3.2, Lemma 3.4. Lemma 3.6 in Section 6.

The detail of the induction procedure, (which is non trivial), that is used to prove Lemma 3.3 in dimension $d=2$ is given in Section 5. We remark here the proof of Lemma 3.3 relies on Lemma 3.2.

Finally, we prove Lemma 3.5 at the end of Section 7 which, together with Lemma 3.6 will conclude the proof of Lemma 3.3 in dimension $d \geq 3$.

\section{Proposition 3.1 implies Theorem 1.3}

We first introduce one standard but important tool in the following lemma.

Lemma 4.1. [4], [5]/ Let $\left\{g_{\alpha}\right\}$ be a family of functions such that supp $\widehat{g}_{\alpha}$ are finitely overlapped cubes of length $\rho$. Let $A$ be bounded convex open set tiled by finitely overlapped cubes $Q$ of side length $\geq \rho^{-1}$, then for the $w_{A}$ adapted to A, the following holds,

$$
f_{A}\left|\sum g_{\alpha}\right|^{2} w_{A} \lesssim \sum \frac{1}{m(A)} \int\left|g_{\alpha}\right|^{2} w_{A}
$$


Proof. Since we can sum up the weight function over a finitely overlapping cover $\{Q\}$ of $A: w_{A}=\sum_{Q \subset A} w_{Q}$, it suffices to prove for $A=Q$. Recall by the inequality 1.15, we cover the whole space $\mathbb{R}^{n}$ by translations $Q^{\prime}$ of $Q$,

$$
\begin{aligned}
f_{Q}\left|\sum g_{\alpha}\right|^{2} w_{Q} d x & \leq \frac{1}{m(Q)} \sum_{Q^{\prime}} \int_{Q^{\prime}} w_{Q}\left(Q^{\prime}\right)\left|\sum g_{\alpha}\right|^{2} \\
& \leq \frac{1}{m(Q)} \sum_{Q^{\prime}} w_{Q}\left(Q^{\prime}\right) \int\left|\sum g_{\alpha}\right|^{2} \mu_{Q^{\prime}}^{2} \\
& =\frac{1}{m(Q)} \sum_{Q^{\prime}} w_{Q}\left(Q^{\prime}\right) \int\left|\widehat{g}_{\alpha} * \widehat{\mu_{Q^{\prime}}}\right|^{2} \\
& \lesssim \frac{1}{m(Q)} \sum_{Q^{\prime}} w_{Q}\left(Q^{\prime}\right) \sum_{\alpha} \int\left|g_{\alpha}\right|^{2} \mu_{Q^{\prime}}^{2} \\
& \lesssim \frac{1}{m(Q)} \sum_{\alpha} \int\left|g_{\alpha}\right|^{2} w_{Q}
\end{aligned}
$$

Now we can reduce Theorem 3.1 to a bilinear decoupling on two $\frac{N_{2}}{N_{1}}$-diameter caps.

Lemma 4.2. Theorem 1.3 is equivalent to Proposition 3.1.

Proof. Let $f_{1}, f_{2}$ be as in Theorem 1.3. Then $f_{1}=\sum_{|\tau|=\frac{N_{2}}{N_{1}}} f_{1, \tau}$ and $f_{1, \tau}$ 's are supported on finitely overlapping caps of diameter $\frac{N_{2}}{N_{1}}$.

Since $\left|f_{2}\right|$ is supported in a cap of diameter $\frac{N_{2}}{N_{1}}$, the supports of $\left\{\widehat{E f}_{1, \tau} *{\widehat{E f_{2}}}_{\tau}\right\}_{\tau}$ are in finitely overlapping cubes of length $\frac{N_{2}}{N_{1}}$. Since the scale of $\Omega$ is larger than $N_{1} / N_{2}$, i.e. it contains a ball of radius $>N_{1} / N_{2}$, By Lemma 4.1,

$$
f_{\Omega}\left|E f_{1} E f_{2}\right|^{2} w_{\Omega} d x \leq \sum_{|\tau|=\frac{N_{2}}{N_{1}}}\left|f_{\Omega} E f_{1, \tau} E f_{2}\right|^{2} w_{\Omega} d x
$$

Now apply Proposition 3.1 for $f_{1, \tau}$ and $f_{2}$ for each $\tau$, Theorem 1.3 follows.

\section{TRAnSVERsality}

Let $f_{1}, f_{2}$ be as in Proposition 3.1 then $f_{1}$ is supported around $(0,0, \ldots, 0,1,1)$ and $f_{2}$ is supported around $(0,0, \ldots, 0)$. The main goal of this section is to explore the transversality between $(0,0, \ldots, 0,1)$ and $(0,0,0, \ldots, 0)$, or more precisely, the transversality between the unit normal vectors of the truncated parabola at these two points. The main lemma in this section is Lemma 5.1 below, and Corollary 5.7 which essentially follows from Lemma 5.1

We first introduce some basic notation. Let $\left(e_{1}, \ldots, e_{d}\right)$ be the standard basis of $\mathbb{R}^{d}$. We will encounter caps of radius $v$ around $(0,0, \ldots, 0)$ and $(0, \ldots, 0,1,1)$ on the parabola. Note around those two points, when $v$ is small (which is always the case in our work), one may view those caps as their natural projection to $\mathbb{R}^{d-1}$. And their image is essentially a square/cap of radius $v$. We say that a $\left(v, v^{2}\right)$-plate is a $d$-dimensional rectangle with the short side on $e_{d-1}$ direction such that its image under under the orthogonal projection to $R^{d-1}$ is a $v \times v \times \cdots \times v \times v^{2}$-rectangle.

Lemma 5.1. Given $|v|<1$, let $f_{1}$ be a function supported on a cap of radius $v$, centered at $(0, \ldots, 0,1,1)$ on the truncated parabola $P$, and let $f_{2}$ be a function supported on a cap of radius $v$ centered at $(0, \ldots, 0,0,0)$ on the paraboloid. For a covering $\left\{\tau_{i}\right\}$ of supp $f_{i}$ with $\left(v, v^{2}\right)$-plates, with the shorter side on $e_{d-1}$ direction. We have the following decoupling inequality, for any $R>v^{-2}$,

$$
\int\left|E f_{1} E f_{2}\right|^{2} w_{B_{R}} \lesssim \sum_{\tau_{1}, \tau_{2}} \int\left|E f_{1, \tau_{1}} E f_{2, \tau_{2}}\right|^{2} w_{B_{R}} .
$$

Remark 5.2. We thank J. Ramos for pointing out that Lemma 5.1 is a particular case of Proposition 2 in his work [15]. We still write a proof in this paper for clarity. 
Proof. The proof is similar to the proof of the $L^{4}$ Strichartz estimate on the one dimensional torus. From the inequality 1.15, we only need to prove that

$$
\int_{B^{\prime}}\left|E f_{1} E f_{2}\right|^{2} \lesssim \sum_{\tau_{1}, \tau_{2}} \int\left|E f_{1, \tau_{1}} E f_{2, \tau_{2}}\right|^{2} \mu_{B^{\prime}}^{2}
$$

for all translation $B^{\prime}$ of $B_{R}$.

$$
\int_{B^{\prime}}\left|E f_{1} E f_{2}\right|^{2} \leq \sum_{\tau_{1}, \tau_{2}, \tau_{3}, \tau_{4}} \int_{B^{\prime}} E f_{1, \tau_{1}} E f_{2, \tau_{2}} \overline{E f_{1, \tau_{3}} E f_{2, \tau_{4}}} \mu_{B^{\prime}}^{2}
$$

Let $\xi_{i} \in \tau_{i}, \xi_{i}=\left(\xi_{i, 1}, . ., \xi_{i, d-1}, \sum_{j=1}^{d-1}\left(\xi_{i}^{j}\right)^{2}\right) \equiv\left(\bar{\xi}_{i}, \xi_{i, d-1},\left|\bar{\xi}_{i}\right|^{2}+\left(\xi_{i}^{d-1}\right)^{2}\right), i=1,2,3,4$. We have

$$
\begin{array}{ll}
\left|\bar{\xi}_{i}\right| \lesssim v, & i=1,2,3,4 . \\
\left|\xi_{i, d-1}-1\right| \lesssim v, & i=1,3 . \\
\left|\xi_{i, d-1}\right| \lesssim v, & i=2,4 .
\end{array}
$$

Essentially, for any $\tau_{1}, \tau_{2}, \tau_{3}, \tau_{4}$ such that

$$
\int E f_{1, \tau_{1}} E f_{2, \tau_{2}} \overline{E f_{1, \tau_{3}} E f_{2, \tau_{4}}} \mu_{B^{\prime}}^{2} \neq 0
$$

one must have for some $\xi_{i} \in \tau_{i}$,

$$
\begin{array}{r}
\xi_{1}-\xi_{3}=\xi_{2}-\xi_{4}+O\left(R^{-1}\right), \\
\left|\xi_{1}\right|^{2}-\left|\xi_{3}\right|^{2}=|\xi|_{2}^{2}-|\xi|_{4}^{2}+O\left(R^{-1}\right),
\end{array}
$$

and the second formula in (5.4) implies

$$
\left(\xi_{1, d-1}-\xi_{3, d-1}\right)\left(\xi_{1, d-1}+\xi_{3, d-1}\right)=O\left(\left|\xi_{2}\right|^{2}+\left|\xi_{4}\right|^{2}\right)+O\left(\left|\bar{\xi}_{1}\right|^{2}+\left|\bar{\xi}_{3}\right|^{2}\right)+O\left(R^{-1}\right) .
$$

Plugging into (5.3), one has $\left|\xi_{1, d-1}-\xi_{3, d-1}\right| \lesssim v^{2}$, which again implies $\left|\xi_{2, d-1}-\xi_{4, d-1}\right| \lesssim v^{2}$.

To summarize, $\int E f_{1, \tau_{1}} E f_{2, \tau_{2}} \overline{E f_{1, \tau_{3}} E f_{2, \tau_{4}}} \mu_{B^{\prime}}^{2} \neq 0$ implies the distance between $\tau_{1}$ and $\tau_{3}$ and the distance between $\tau_{2}$ and $\tau_{4}$ are both bounded by $v^{2}$, which essentially means $\tau_{i}=\tau_{i+2}, i=1,2$. Applying this fact to (5.2), Lemma 5.1 follows.

Remark 5.3. A quantitative version of estimate (5.1) can be stated as follows: assume that the support of $f_{1}$ is centered at $\left(0,1 / K,(1 / K)^{2}\right)$ rather than $(0,0,1)$, from the proof we can attain the same estimate as in (5.1) by introducing an additional constant $K$,

$$
\int\left|E f_{1} E f_{2}\right|^{2} w_{B_{R}} \lesssim K \sum_{\tau_{1}, \tau_{2}} \int\left|E f_{1, \tau_{1}} E f_{2, \tau_{2}}\right|^{2} w_{B_{R}}
$$

Indeed, the proof essentially only relies on the fact that for $\xi_{i} \in$ supp $f_{i}, i=1,2$, the difference between the $d-1$ components is at least $\frac{1}{K}$. Similar arguments also hold for estimate in Lemma [5.5. Cor 5.7 below.

Remark 5.4. We remark that for any $\alpha<v$, a function which is supported on a cap of radius $\alpha$ can be naturally understood as a function supported on a cap of radius $v$.

Lemma 5.1 facilitates the decomposition of caps of radius $v$ into plates of size $\left(v, v^{2}\right)$, we can further decompose those into caps of radius $v^{2}$.

Lemma 5.5. With same notation as in Lemma 5.1, $R \geq v^{-2}$, let supp $f_{i}$ be the covered by finitely overlapping caps $\theta_{i}$ of radius $v^{2}, i=1,2$. Then

$$
\int\left|E f_{1} E f_{2}\right|^{2} w_{B_{R}} \lesssim v^{-(d-1)} \sum_{\left|\theta_{i}\right|=v^{2}} \int\left|E f_{1, \theta_{1}} E f_{2, \theta_{2}}\right|^{2} w_{B_{R}} .
$$

Proof. Clearly, we need only to prove (5.7) for every ball of radius $v^{-2}$ contained in $B_{R}$, and then sum them together. (This is in the same principle of parallel decoupling, Lemma 1.9]) 
Fix a pair of $\left(v, v^{2}\right)$-plates $\tau_{1}, \tau_{2}$.

$$
\begin{aligned}
\int\left|E f_{1, \tau_{1}} E f_{2, \tau_{2}}\right|^{2} w_{B_{R}} & =\int\left|\sum_{\theta_{2} \subset \tau_{2},\left|\theta_{2}\right|=v^{2}} E f_{1, \tau_{1}} E f_{2, \theta_{2}}\right|^{2} w_{B_{R}} \\
& \leq v^{-(d-1)} \sum_{\theta_{2} \subset \tau_{2},\left|\theta_{2}\right|=v^{2}}\left|E f_{1, \tau_{1}} E f_{2, \theta_{2}}\right|^{2} w_{B_{R}} \\
& \lesssim \sum_{\theta_{j} \subset \tau_{j},\left|\theta_{j}\right|=v^{2}}\left|E f_{1, \theta_{1}} E f_{2, \theta_{2}}\right|^{2} w_{B_{R}}
\end{aligned}
$$

The last inequality follows from Lemma 4.1 and Lemma 4.2 ,

Remark 5.6. Similar to Remark 5.4, for $v^{2}<\alpha<v$, a cap of scale $v$ naturally lies in a cap of scale $\sqrt{\alpha}$. Thus if we let $f_{1}$ be a function supported on a cap of radius $\alpha$, centered at $(0, \ldots, 0,1,1)$ on the paraboloid and we let $f_{2}$ be a function supported on a cap of radius $\alpha$ centered at $(0, \ldots, 0,0,0)$ on the paraboloid, then by arguing similar to the proof of Lemma 5.5, we have for $R \geq \alpha^{-1}$,

$$
\int\left|E f_{1} E f_{2}\right|^{2} w_{B_{R}} \lesssim(v / \alpha)^{(d-1)} \sum_{\left|\theta_{i}\right|=\alpha} \int\left|E f_{1, \theta_{1}} E f_{2, \theta_{2}}\right|^{2} w_{B_{R}} .
$$

If we directly use Holder inequality for all caps in the support of $f_{i}$ to estimate as in (5.8), then the interpolation in the proof of Lemma 5.5 will give us a constant $v^{-d}$ rather than $v^{-(d-1)}$ in (5.7), since one has $v^{-d}$ caps for each $f_{i}$. The bilinear transversality, i.e. the transversality between $(0,0, \ldots, 0)$ and $(0, \ldots, 0,1,1)$ helps in reducing the dimension by one since in one direction we can use $L^{4}$ orthogonality, as shown in Lemma [5.1. Thus here we are able to improve the constant in (5.7) to $v^{-(d-1)}$.

Corollary 5.7. Same notation as in Lemma 5.1, there exists a constant $C$, such that for any $v, \delta, R^{-1} \leq \delta \leq v$,

$$
\int\left|E f_{1} E f_{2}\right|^{2} w_{B_{R}} \lesssim\left(\frac{v}{\delta}\right)^{d-1}\left|\frac{\log \delta}{\log v}\right|^{C} \sum_{\left|\theta_{i}\right|=\delta} \int\left|E f_{1, \theta_{1}} E f_{2, \theta_{2}}\right|^{2} w_{B_{R}} .
$$

Proof. The proof is most clear when $\delta=v^{2^{n}}$ for some $n$, let us first handle this case and then go to the general case. One may use induction. (This induction, however, does not rely on parabolic rescaling.) If $n=0$, there is nothing to prove.

Assume the result holds for the case $n=k$, let us turn to the case $n=k+1$, where $\delta=v^{2^{k+1}}, \delta^{1 / 2}=v^{2^{k}}$, thus by induction assumption, we have

$$
\int\left|E f_{1} E f_{2}\right|^{2} w_{B_{R}} \lesssim\left(\frac{v}{\delta^{1 / 2}}\right)^{d-1} 2^{C k} \sum_{\left|\eta_{i}\right|=\delta^{1 / 2}} \int\left|E f_{1, \eta_{1}} E f_{2, \eta_{2}}\right|^{2} w_{B_{R}} .
$$

Now note $R \geq\left(\delta^{-1 / 2}\right)^{2}$, by Lemma 5.5, we have for each pair $\left(\eta_{1}, \eta_{2}\right)$ in (5.10) that

$$
\int\left|E f_{1, \eta_{1}} E f_{2, \eta_{2}}\right|^{2} w_{B_{R}} \lesssim\left(\delta^{1 / 2}\right)^{-(d-1)} \sum_{\theta_{i} \subset \eta_{i},\left|\theta_{i}\right|=\delta} \int\left|E f_{1, \theta_{1}} E f_{2, \theta_{2}}\right|^{2} w_{B_{R}} .
$$

The case $n=k+1$ clearly follows if one plugs (5.11) into (5.10), taking the constant $C$ large enough.

Now we turn to the general case, we only need to work on the case $v^{2^{n+1}}<\delta<v^{2^{n}}$. Recall that previously, when $\delta=v^{2^{n}}$, we used induction as $v \rightarrow v^{2} \rightarrow v^{2^{2}} \cdots \rightarrow v^{2^{n}}=\delta$, and in each step we used Lemma 5.5 to finish the induction $v^{2^{k}} \rightarrow v^{2^{k+1}}$.

In the case $v^{2^{n+1}}<\delta<v^{2^{n}}$ we have $v^{2^{n}}<\delta^{1 / 2}$, and we use induction as before for $v \rightarrow v^{2} \rightarrow v^{2^{2}} \cdots \rightarrow v^{2^{n}}$, and we use (5.9) to use induction again from $v^{2^{n}}$ to $\delta$. This ends the proof.

\section{Proof of Lemma 3.2, Lemma 3.4 and Lemma 3.6}

We are now prepared to use transversality to prove Lemma 3.2, Lemma 3.4, and Lemma 3.6, Recall Lemma 3.2 concerns $\tilde{K}\left(\lambda, N_{1}, N_{2}\right)$ defined in (3.4). Furthermore, Lemma 3.4 and Lemma 3.6 refer to $K\left(\lambda, N_{1}, N_{2}\right)$ defined in (3.5). 
6.1. Proof of Lemma 3.2. For convenience of notation, we let $\Omega_{1}:=\left[0, N_{1}^{2}\right] \times\left[0, \lambda N_{1}\right]^{d}$. Note that one can use finite overlapped balls of radius $N_{1}^{2}$ to cover $\Omega_{1}$ since $\lambda \geq N_{1}$. We want to prove

$$
\left\|E f_{1} E f_{2}\right\|_{L_{a v g}^{2}\left(\omega_{\Omega_{1}}\right)} \lesssim_{\epsilon} \lambda^{d / 2} N_{2}^{\epsilon} \frac{N_{2}^{d-1}}{N_{1}} \prod_{j=1}^{2}\left(\sum_{|\theta|=\frac{1}{\lambda N_{1}}}\left\|E f_{j, \theta}\right\|_{L_{a v g}^{4}\left(w_{\Omega_{1}}\right)}^{2}\right)^{1 / 2} .
$$

We first apply Corollary 5.7 with $\delta=N_{1}^{-2}, v=\frac{N_{2}}{N_{1}}, R=N_{1}^{2}$. Note that $\delta \leq v$. Then we have

$$
\begin{aligned}
\int\left|E f_{1} E f_{2}\right|^{2} w_{B_{N_{1}^{2}}} & \lesssim\left(N_{1} N_{2}\right)^{d-1}\left|\frac{\log N_{1}}{\log N_{1}-\log N_{2}}\right|^{C} \sum_{\left|\theta_{j}\right|=\frac{1}{N_{1}^{2}}} \int\left|E f_{1, \theta_{1}} E f_{2, \theta_{2}}\right|^{2} w_{B_{N_{1}^{2}}} \\
& \lesssim\left(N_{1} N_{2}\right)^{d-1} N_{2}^{\epsilon} \sum_{\left|\theta_{j}\right|=\frac{1}{N_{1}^{2}}} \prod_{j=1}^{2}\left\|E f_{j, \theta_{j}}\right\|_{L^{4}\left(w_{B_{N_{1}}}{ }^{2}\right.}^{2} .
\end{aligned}
$$

Remark 6.1. We avoid the case when $N_{1}=N_{2}$, and thus $\ln N_{1}-\ln N_{2}=0$, by first decomposing caps of diameter $N_{2} / N_{1}$ into caps of diameter $N_{2} / 2 N_{1}$ with loss of a fixed constant, then continuing with the proof as above. In all of the text that follows, one may assume, without loss of generality, that $N_{1} \geq 2 N_{2}$.

Via the principle of parallel decoupling, Lemma [1.9] or by summing different $B_{N_{1}^{2}}$ together, we have

$$
\int\left|E f_{1} E f_{2}\right|^{2} w_{\Omega_{1}} \lesssim\left(N_{1} N_{2}\right)^{d-1} N_{2}^{\epsilon} \sum_{\left|\theta_{j}\right|=\frac{1}{N_{1}^{2}}} \prod_{j=1}^{2}\left\|E f_{j, \theta_{j}}\right\|_{L^{4}\left(w_{\Omega_{1}}\right)}^{2} .
$$

Next we would like to show that

$$
\left\|E f_{j, \theta_{j}}\right\|_{L^{4}\left(w_{\Omega_{1}}\right)}^{2} \leq\left(\frac{\lambda}{N_{1}}\right)^{d / 2} \sum_{\theta_{j}^{\prime} \subset \theta_{j},\left|\theta_{j}^{\prime}\right|=\frac{1}{\lambda N_{1}}}\left\|E f_{j, \theta_{j}^{\prime}}\right\|_{L^{4}\left(w_{\Omega_{1}}\right)}^{2} .
$$

It suffices to show

$$
\left\|E f_{j, \theta_{j}}\right\|_{L_{a v g}^{4}\left(\Omega_{1}\right)}^{2} \leq\left(\frac{\lambda}{N_{1}}\right)^{d / 2} \sum_{\theta_{j}^{\prime} \subset \theta_{j},\left|\theta_{j}^{\prime}\right|=\frac{1}{\lambda N_{1}}}\left\|E f_{j, \theta_{j}^{\prime}}\right\|_{L_{a v g}^{4}\left(w_{\Omega_{1}}\right)}^{2}
$$

and sum up as in Lemma 4.1

Each function $E f_{j, \theta_{j}^{\prime}}$ is fourier supported in $\theta_{j}^{\prime}$, in particular, fourier supported in a cylinder of radius $\frac{1}{\lambda N_{1}}$, height $\frac{1}{N_{1}^{2}}$. $\Omega_{1}$ is tiled by cylinders of radius $\lambda N_{1}$, height $N_{1}^{2}$ in $t$-direction. The proof of Lemma 4.1 works the same,

$$
\begin{aligned}
\left\|E f_{j, \theta_{j}}\right\|_{L_{a v g}^{2}\left(\Omega_{1}\right)}^{2} & \sum_{\theta_{j}^{\prime} \subset \theta_{j},\left|\theta_{j}^{\prime}\right|=\frac{1}{\lambda N_{1}}}\left\|E f_{j, \theta_{j}^{\prime}}\right\|_{L_{a v g}^{2}\left(w_{B_{R}}\right)}^{2}\left\|E f_{j, \theta_{j}^{\prime}}\right\|_{L_{a v g}^{4}\left(w_{B_{R}}\right)}^{2} \\
& \lesssim \sum_{\theta_{j}^{\prime} \subset \theta_{j},\left|\theta_{j}^{\prime}\right|=\frac{1}{\lambda N_{1}}}
\end{aligned}
$$

For the $L^{\infty}$-estimate, we apply Cauchy Schwartz inequality:

$$
\begin{aligned}
\left\|E f_{j, \theta_{j}}\right\|_{L^{\infty}\left(\Omega_{1}\right)}^{2} & \leq\left(\frac{\lambda}{N_{1}}\right)^{d} \sum_{\theta_{j}^{\prime} \subset \theta_{j},\left|\theta_{j}^{\prime}\right|=\frac{1}{\lambda N_{1}}}\left\|E f_{j, \theta_{j}^{\prime}}\right\|_{L^{\infty}\left(\Omega_{1}\right)}^{2} \\
& \lesssim\left(\frac{\lambda}{N_{1}}\right)^{d} \sum_{\theta_{j}^{\prime} \subset \theta_{j},\left|\theta_{j}^{\prime}\right|=\frac{1}{\lambda N_{1}}}\left\|E f_{j, \theta_{j}^{\prime}}\right\|_{L_{a v g}^{4}\left(w_{\Omega_{1}}\right)}^{2}
\end{aligned}
$$

The last inequality is an application of Lemma 1.10. Note $f_{\theta_{j}^{\prime}}$ is supported in a ball of scale $\frac{1}{\lambda N_{1}}$, and inside a box $C$ of size $\frac{1}{N_{1}^{2}} \times \frac{1}{\lambda N_{1}} \times \cdots \times \frac{1}{\lambda N_{1}}$. We can make a affine transform of $C$ into a cube $Q^{*}$ of scale $\lambda_{N_{1}}$, which on the physical side would transform $\Omega_{1}$ into a cube of scale $\lambda N_{1}$. We apply Lemma 1.10 after the affine transformation and then transform back. (Note in those setting, cube is no different than a ball.)

We apply Hölder's inequality to conclude the argument. 
6.2. Proof of Lemma 3.4. Let $\lambda \leq N_{1}$. We first note that we can use finitely overlapping balls $B_{\lambda N_{1}}$ to cover $\Omega$ and that $N_{2} \lesssim 1$. Applying Corollary [5.7 with $\delta=\frac{1}{\lambda N_{1}}$ and $v=\frac{N_{2}}{N_{1}}$ we have

$$
\begin{aligned}
\int\left|E f_{1} E f_{2}\right|^{2} w_{B_{\lambda N_{1}}} & \lesssim\left(\lambda N_{2}\right)^{d-1}\left|\frac{\log \lambda+\log N_{1}}{\log N_{1}-\log N_{2}}\right|^{C} \sum_{\left|\theta_{j}\right|=\frac{1}{\lambda N_{1}}} \int\left|E f_{1, \theta_{1}} E f_{2, \theta_{2}}\right|^{2} w_{B_{\lambda N_{1}}} \\
& \lesssim\left(\lambda N_{2}\right)^{d-1} N_{2}^{\epsilon} \sum_{\left|\theta_{j}\right|=\frac{1}{\lambda N_{1}}} \prod_{j=1}^{2}\left\|E f_{j, \theta_{j}}\right\|_{L^{4}\left(w_{B_{\lambda N_{1}}}\right)}^{2} .
\end{aligned}
$$

With parallel decoupling, Lemma 1.9, then the desired estimate follows. (As remarked in Remark 6.1, one can assume $N_{1} \geq 2 N_{2}$.)

6.3. Proof of Lemma 3.6. Let $\lambda \leq N_{1}$.

We have the following two cases:

- Case 1: $d=2, N_{1} \geq \lambda \geq \frac{N_{1}}{N_{2}}$, and $N_{2}^{\prime}=\left(\frac{N_{1}}{\lambda}\right)$,

- Case 2: $d \geq 3, N_{1} \geq \lambda \geq \frac{N_{1}}{N_{2}^{2}}$, and $N_{2}^{\prime}=\left(\frac{N_{1}}{\lambda}\right)^{1 / 2}$.

It is easy to check that we only need to show that

$$
K\left(\lambda, N_{1}, N_{2}\right) \lesssim K\left(\lambda, N_{1}, N_{2}^{\prime}\right)\left(\frac{N_{1}}{N_{2}^{\prime}} \frac{N_{2}}{N_{1}}\right)^{\frac{d-1}{2}} .
$$

We claim that

$$
\left\|E f_{1} E f_{2}\right\|_{L_{a v g}^{4}\left(w_{B_{N_{1}^{2}}}\right)} \lesssim\left(\frac{N_{2} / N_{1}}{N_{2}^{\prime} / N_{1}}\right)^{d-1 / 2} \prod_{j=1}^{2}\left(\sum_{|\theta|=\frac{N_{2}^{\prime}}{N_{1}}}\left\|E f_{j, \theta}\right\|_{L_{a v g}^{4}\left(w_{B_{N}}^{2}\right)}^{2}\right)^{1 / 2}
$$

Since $\lambda \leq N_{1}$, we cover $B_{N_{1}^{2}}$ with balls of radius $\lambda N_{1}$. Thus by parallel decoupling, to prove (6.6), we only need to show

$$
\left\|E f_{1} E f_{2}\right\|_{L_{a v g}^{4}\left(w_{B_{\lambda N_{1}}}\right)} \lesssim\left(\frac{N_{2} / N_{1}}{N_{2}^{\prime} / N_{1}}\right)^{d-1 / 2} \prod_{j=1}^{2}\left(\sum_{|\theta|=\frac{1}{\lambda N_{1}}}\left\|E f_{j, \theta}\right\|_{L_{a v g}^{4}\left(w_{B_{\lambda N_{1}}}\right)}^{2}\right)^{1 / 2} .
$$

Note that since $\lambda N_{1} \geq \frac{1}{N_{2}^{\prime} / N_{1}}$, estimate (6.7) follows from Corollary (5.7) by setting $\delta=N_{2} / N_{1}, v=N_{2}^{\prime} / N_{1}$ via interpolation and local constant arguments as in Section 6.1

By the definition of $K\left(\lambda, N_{1}, N_{2}\right)$, we have that for any $\theta_{1}, \theta_{2}$ in (6.7),

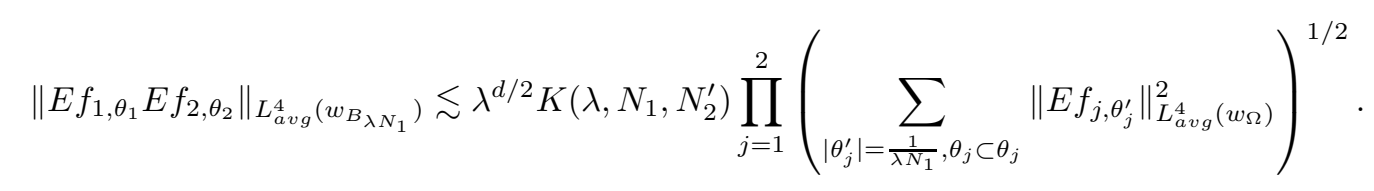

Plugging (6.8) into (6.7), clearly (6.5) follows.

\section{IndUCtion PROCEDURE AND PROOF OF LEMma 3.3}

To conclude the proof of Proposition 3.1, we are left with the proof of Lemma 3.3. For this lemma the proof relies on induction on $N_{2}$. The base case $N_{2} \lesssim 1$ is resolved by Lemma 3.4 and by Lemma 3.6, so we need only to induct until $\lambda=\frac{\left(N_{2}\right)^{d-1}}{N_{1}}$.

Let $f_{1}, f_{2}$ be as in Lemma 3.3. Applying Lemma 5.1, taking $v=N_{1} / N_{2}$ and $R=N_{1}^{2}$, we could decouple the $\frac{N_{2}}{N_{1}}$ caps into $\left(\frac{N_{2}}{N_{1}}, \frac{N_{2}^{2}}{N_{1}^{2}}\right)$ plates without any loss, i.e.

$$
\int\left|E f_{1} E f_{2}\right|^{2} w_{B_{N_{1}^{2}}} \lesssim \sum_{\tau_{1}, \tau_{2}} \int\left|E f_{1, \tau_{1}} E f_{2, \tau_{2}}\right|^{2} w_{B_{N_{1}}^{2}}
$$

Here $\tau_{i}$ are plates as described in Lemma 5.1. We focus on the case when $d=2$ in $\mathbb{R}^{3}$, the high dimensional case would be explained in the end. When $d=2$, the underlying plates become strips. We start with some preparation before the induction. 
7.1. Preliminary preparation for the induction. We fix a pair of $\left(\frac{N_{2}}{N_{1}}, \frac{N_{2}^{2}}{N_{1}^{2}}\right)$ strips $\tau_{1}, \tau_{2}$ from estimate (7.1) . We decompose $\tau_{j}$ into a union of $\frac{N_{2}}{K N_{1}} \times \frac{N_{2}^{2}}{N_{1}^{2}}$ strips $\left\{s_{j}\right\}$.

Using the notation nonadj short for nonadjacent, and adj short for adjacent, we have

$$
\begin{aligned}
\left|E f_{\tau_{j}}\right|^{2} & =\sum_{s_{j}}\left|E f_{s_{j}}\right|^{2}+\sum_{s_{j}, s_{j}^{\prime} a d j}\left|E f_{s_{j}} E f_{s_{j}^{\prime}}\right|+\sum_{s_{j}, s_{j}^{\prime} \text { nonadj }}\left|E f_{s_{j}} E f_{s_{j}^{\prime}}\right| \\
& \leq 10 \sum_{s_{j}}\left|E f_{s_{j}}\right|^{2}+\sum_{s_{j}, s_{j}^{\prime} \text { nonadj }}\left|E f_{s_{j}} E f_{s_{j}^{\prime}}\right| \\
& =I_{j, 1}+I_{j, 2} \\
\int\left|E f_{\tau_{1}} E f_{\tau_{2}}\right|^{2} w_{B_{N_{1}^{2}}} & \leq \int\left|\left(E f_{\tau_{1}}^{2}-I_{1,1}\right)\left(\mid E f_{\tau_{2}}^{2}-I_{2,1}\right)\right|+E f_{\tau_{1}}^{2} I_{2,1}+E f_{\tau_{2}}^{2} I_{1,1}+I_{1,1} I_{2,1} w_{B_{N_{1}^{2}}} \\
& \lesssim \sum_{s_{j}, s_{j}^{\prime} \text { nonadj }} \int\left|E f_{s_{1}} E f_{s_{1}^{\prime}} E f_{s_{2}} E f_{s_{2}^{\prime}}\right| w_{B_{N_{1}^{2}}}+\sum_{s_{1}, s_{2}} \int\left|E f_{s_{1}} E f_{s_{2}}\right|^{2} w_{B_{N_{1}^{2}}}
\end{aligned}
$$

The last inequality follows from Lemma 4.1 and Lemma 4.2 .

The reason why we want to have non-adjacent parts is that we would like transversality (after rescaling) on the other direction. Formula (7.3) will the starting point of our induction.

For the second term in (7.3), we will later directly use induction ( not relying on parallel rescaling) on $N_{2}$ and reduce everything to the known base case $N_{2}=1$.

For the first term, using Cauchy-Schwartz

$$
\int\left|E f_{s_{1}} E f_{s_{1}^{\prime}} E f_{s_{2}} E f_{s_{2}^{\prime}}\right| w_{B_{N_{1}^{2}}} \leq\left(\int\left|E f_{s_{1}} E f_{s_{1}^{\prime}}\right|^{2} w_{B_{N_{1}^{2}}}\right)^{1 / 2}\left(\int\left|E f_{s_{2}} E f_{s_{2}^{\prime}}\right|^{2} w_{B_{N_{1}^{2}}}\right)^{1 / 2} .
$$

We point out here that in what follows we do not rely on the bilinear transversality between $s_{1}$ and $s_{2}$ (or $s_{1}$ and $s_{2}^{\prime}$ ), which is already handled in Lemma 5.1. Instead we will rely on the bilinear transversality between $s_{1}$ and $s_{1}^{\prime}$, (or $s_{2}, s_{2}^{\prime}$ ), since they are not adjacent. This transversality is most clear when one applies parabolic rescaling.

Let us now turn to the term $\int\left|E f_{s_{2}} E f_{s_{2}^{\prime}}\right|^{2} w_{\Omega}$, when $s_{2}, s_{2}^{\prime}$ are non adjacent. The term with $s_{1}, s_{1}^{\prime}$ is handled similarly, though one may need to rotate the coordinates.

Finally we point out here that $K$ would be chosen large later and any (fixed) power of $K$ will not impact the final estimate. In particular, in the following estimates we would not worry about losing powers of $K$.

Without loss of generality, we assume

- $s_{2}$ is the strip that $\left\{\left(a_{1}, a_{2}, a_{1}^{2}+a_{2}^{2}\right)|| a_{1}\left|\leq N_{2}^{2} / N_{1}^{2},\right| a_{2} \mid \leq N_{2} / K N_{1}\right\}$

- $s_{2}^{\prime}$ is the strip that $\left\{\left(b_{1}, b_{2}, b_{1}^{2}+b_{2}^{2}\right)|| b_{1}\left|\leq N_{2}^{2} / N_{1}^{2},\right| b_{2}-C N_{2} / K N_{1} \mid \leq N_{2} / K N_{1}\right\}, C \geq 10$. (Here 10 is of course just some universal constant.)

7.2. Parabolic rescaling. The next step, parabolic scaling, is standard in decoupling types results; we give the details here for the convenience of the reader.

Note $s_{2}, s_{2}^{\prime}$ lie on the same $\frac{N_{2}}{N_{1}}$ cap. We rescale the $\frac{N_{2}}{N_{1}}$ cap to radius 1 . By a slight abuse of notation, we regard $f_{s_{i}}$ as a function depending only on two variables $\left(\xi_{i, 1}, \xi_{i, 2}\right)$. For convenience notation, we let $h_{1}=f_{s_{2}}, h_{2}=f_{s_{2}^{\prime}}$. Let also $g_{i}\left(\eta_{i, 1}, \eta_{i, 2}\right):=h_{i}\left(\left(N_{2} / N_{1}\right) \eta_{i, 1},\left(N_{2} / N_{1}\right) \eta_{i, 2}\right)$.

Note now

- $g_{1}$ is supported in the strip of $\left\{\left(a_{1}, a_{2}, a_{1}^{2}+a_{2}^{2}\right)|| a_{1}\left|\leq N_{2} / N_{1},\right| a_{2} \mid \leq 1 / K\right\}$

- $g_{2}$ is supported in the strip of $\left\{\left(b_{1}, b_{2}, b_{1}^{2}+b_{2}^{2}\right)|| b_{1}\left|\leq N_{2} / N_{1},\right| b_{2}-C / K \mid \leq 1 / K\right\}, C \geq 10$

Note $g_{1}, g_{2}$ are supported on a pair of transverse $\frac{N_{2}}{N_{1}} \times 1$ strip\& 1 due to the non adjacency of $s_{2}, s_{2}^{\prime}$. We point out here the transversality between $g_{1}, g_{2}$ is not as in the assumption of Lemma [5.1, but it is in the sense of Remark 5.3. which usually cause a loss of $K$ in the estimate, but this does not matter.

The parabolic scaling says the following:

\footnotetext{
${ }^{1}$ Strictly speaking, we need them to support on a pair of $\frac{N_{2}}{N_{1}} \times \frac{1}{100}$ strips, we neglect this technical point here.
} 
Claim 7.1. Let $E g_{i}\left(y_{1}, y_{2}, y_{3}\right)=E h_{i}\left(N_{1} / N_{2} y_{1}, N_{1} / N_{2} y_{2}, N_{1}^{2} / N_{2}^{2} y_{3}\right)$, and let $D$ be domain in $\mathbb{R}^{3}$ and let

$$
\tilde{D}:=\left\{\left(y_{1}, y_{2}, y_{3}\right): N_{1} / N_{2} y_{1}, N_{1} / N_{2} y_{2}, N_{1}^{2} / N_{2}^{2} y_{3} \in D\right\},
$$

then it follows from standard change of variables technique that the following two estimates, with the same constant $A$, are equivalent:

$$
\begin{aligned}
\left\|E h_{1} E h_{2}\right\|_{L_{\text {avg }}^{2}\left(w_{D}\right)} \lesssim A \prod_{j=1}^{2}\left(\sum_{|\theta|=\frac{1}{\lambda N_{1}}}\left\|E f_{s_{j}, \theta}\right\|_{L_{a v g}^{4}\left(w_{D}\right)}^{2}\right)^{1 / 2}, \\
\left\|E g_{1} E g_{2}\right\|_{L_{\text {avg }}^{2}\left(w_{\tilde{D}}\right)} \lesssim A \prod_{j=1}^{2}\left(\sum_{|\tilde{\theta}|=\frac{1}{\lambda N_{2}}}\left\|E g_{j, \tilde{\theta}}\right\|_{L_{\text {avg }}^{4}\left(w_{\tilde{D}}\right)}^{2}\right)^{1 / 2} .
\end{aligned}
$$

we then concentrate on (7.6).

Take $D=B_{N_{1}^{2}}$, then $\tilde{D}=\left[0, N_{2}^{2}\right] \times\left[0, N_{1} N_{2}\right]^{2}$. (Here, without loss of generality, we regard $B_{N_{1}^{2}}$ as $\left[0, N_{1}^{2}\right]^{3}$.) For convenience of notation, we set $\tilde{\Omega}=\left[0, N_{2}^{2}\right] \times\left[0, N_{1} N_{2}\right]^{2}$. The parabolic rescaling gives

Lemma 7.2. Assume $g_{1}, g_{2}$ are two general functions defined on the parabola. Let $g_{1}$ be supported in a strip of size $N_{2} / N_{1} \times 1$ around $(0,0,0)$, and $g_{2}$ be supported in a strip of size $N_{2} / N_{1} \times 1$ around $(0,1,1)$. If for some constant $A$, one has (for all such $g_{1}, g_{2}$ ),

$$
\left\|E g_{1} E g_{2}\right\|_{L_{a v g}^{2}\left(w_{\tilde{\Omega}}\right)} \lesssim A\left(\sum_{|\tilde{\theta}|=\frac{1}{\lambda N_{2}}}\left\|E g_{j, \tilde{\theta}}\right\|_{L_{a v g}^{4}\left(w_{\hat{\Omega}}\right)}^{2}\right)^{1 / 2}
$$

then for the same constant $A$, one has

$$
\left\|E f_{s_{2}} E f_{s_{2}^{\prime}}\right\|_{L_{\text {avg }}^{2}\left(w_{B_{N_{1}^{2}}}\right)} \lesssim K^{C} A\left(\sum_{|\theta|=\frac{1}{\lambda N_{1}}}\left\|E f_{s_{2}, \theta}\right\|_{L_{\text {avg }}^{4}\left(w_{B_{N_{1}^{2}}}\right)}^{2}\right)^{1 / 2}\left(\sum_{|\theta|=\frac{1}{\lambda N_{1}}}\left\|E f_{s_{2}^{\prime}, \theta}\right\|_{L_{\text {avg }}^{4}\left(w_{B_{N}}^{2}\right)}^{2}\right)^{1 / 2} .
$$

Remark 7.3. After rescaling, the relevant $g_{1}, g_{2}$ should be supported around $(0,0,0)$ and $\left(0,1 / K, 1 / K^{2}\right)$ rather than $(0,0,0)$ and $(0,0,1)$. We state our lemma for $g_{1}, g_{2}$ supported around $(0,0,0)$ and $(0,1,1)$ to be consistent with the statement in Lemma [5.1. This causes a loss of $K^{C}$, but we emphasize again that any loss due to a power of $K$ would be irrelevant in the proof.

We end this section by introducing some notation.

Let $g_{1}, g_{2}$ be as in Lemma 7.2, we define $A\left(\lambda, N_{1}, N_{2}\right)$ to be the best constant such that

$$
\left\|E g_{1} E g_{2}\right\|_{L_{\text {avg }}^{2}\left(w_{\tilde{\Omega}}\right)} \lesssim A\left(\lambda, N_{1}, N_{2}\right)\left(\sum_{|\tilde{\theta}|=\frac{1}{\lambda N_{2}}}\left\|E g_{j, \tilde{\theta}}\right\|_{L_{\text {avg }}^{4}\left(w_{\hat{\Omega}}\right)}^{2}\right)^{1 / 2}
$$

Then we can restate Lemma (7.2) .

Lemma 7.4. For $j=1,2$, we have

$$
\begin{aligned}
&\left\|E f_{s_{j}} E f_{s_{j}^{\prime}}\right\|_{L_{a v g}^{2}\left(w_{B_{N_{1}^{2}}}\right)} \\
& \lesssim K^{C} A\left(\lambda, N_{1}, N_{2}\right)\left(\sum_{|\theta|=\frac{1}{\lambda N_{1}}}\left\|E f_{s_{j}, \theta}\right\|_{L_{\text {avg }}^{4}\left(w_{B_{N_{1}^{2}}}\right)}^{2}\right)^{1 / 2}\left(\sum_{|\theta|=\frac{1}{\lambda N_{1}}}\left\|E f_{s_{j}^{\prime}, \theta}\right\|_{L_{\text {avg }}^{4}\left(w_{B_{N_{1}^{2}}}\right)}^{2}\right)^{1 / 2} .
\end{aligned}
$$

\subsection{The induction procedure.}


7.3.1. Before induction. Now we are ready to start the induction for the proof of Lemma 3.3 We emphasize here the induction is on $N_{2}$, (though mixed with induction on $K$ ). Note we are now in dimension $d=2$.

We need to show that for all $1 \leq N_{2} \leq N_{1}$ and $\lambda \leq N_{1}$, one has

$$
K\left(\lambda, N_{1}, N_{2}\right) \lesssim N_{2}^{\epsilon}\left(\frac{1}{\lambda}+\frac{N_{2}}{N_{1}}\right)^{1 / 2}
$$

Note the base case $N_{2}=1$ is already established in Corollary 3.4. And with Lemma 3.6, we need only to perform induction until $\lambda=N_{2} / N_{1}$.

We will work on $A\left(\lambda, N_{1}, N_{2}\right)$ defined in (7.9) to explore the transversality between nonadjacent strips. The induction process is two fold in some sense. We will induct on $N_{2}$ to better understand $K\left(\lambda, N_{1}, N_{2}\right)$, and in turn we find more information about $A\left(\lambda, N_{1}, N_{2}\right)$, which in turn gives a better understanding of $K\left(\lambda, N_{1}, N_{2}\right)$.

This is a final summary before we start the induction. Recall, we have (7.1) and (7.3), thus we have

$$
\begin{aligned}
& \int\left|E f_{1} E f_{2}\right|^{2} w_{B_{N_{1}^{2}}} \\
\lesssim & \int \sum_{s_{j}, s_{j}^{\prime} \text { nonadj }} \int\left|E f_{s_{1}} E f_{s_{1}^{\prime}} E f_{s_{2}} E f_{s_{2}^{\prime}}\right| w_{B_{N_{1}^{2}}} \\
+ & \int_{s_{1}, s_{2}} \int\left|E f_{s_{1}} E f_{s_{2}}\right| w_{B_{N_{1}^{2}}} .
\end{aligned}
$$

Also recall that $s_{1}, s_{1}^{\prime}, s_{2}, s_{2}^{\prime}$ are all $\left(N_{2} / N_{1}\right)^{2} \times N_{2} / K N_{1}$ strips. The second term can be easily handled by direct induction, (which is not the main point of the induction procedure explained later). Indeed, if there were only the second term in (7.11), since $s_{1}, s_{2}$ are both contained in caps of radius $\left(N_{2} / K N_{1}\right)$, then (7.11) already reduces the decoupling problem for $f_{i}$ supported in caps of size $N_{2} / N_{1}$ into the decoupling problem for $f_{i}$ supported in caps of size $N_{2} / K N_{1}$, which reduce $N_{2}$ to $N_{2} / K$.

We will focus on the first term of (7.11). Hölder inequality gives

$$
\int\left|E f_{s_{1}} E f_{s_{1}^{\prime}} E f_{s_{2}} E f_{s_{2}^{\prime}}\right| w_{B_{N_{1}^{2}}} \leq \prod_{j=1}^{2}\left(\int\left|E f_{s_{j}} E f_{s_{j}^{\prime}}\right|^{2} w_{B_{N_{1}^{2}}}\right)^{1 / 2} .
$$

Estimate (7.12) is the start point of the analysis in the following Subsections.

We summarize in the lemma below how (7.12) and (7.11) come together to highlight the relevance of $A\left(N_{1}, N_{2}, \lambda\right)$ in the induction procedure.

Lemma 7.5. When $\lambda \leq N_{1} / N_{2}$ and $\lambda \leq N_{1}$, we have

$$
K\left(N_{1}, N_{2}, \lambda\right) \lesssim K^{C} \frac{1}{\lambda} A\left(N_{1}, N_{2}, \lambda\right)+K\left(N_{1}, N_{2} / K, \lambda\right)
$$

Note that the assumption of Lemma 7.5 always holds during the induction procedure to prove Lemma 3.3

Proof of Lemma 7.5. Applying Lemma 7.4, we have

$$
\begin{aligned}
& \left.\left\|E f_{s_{j}} E f_{s_{j}^{\prime}}\right\|_{L_{a v g}^{2}\left(w_{B} N_{1}^{2}\right.}\right) \\
\lesssim & K^{C} A\left(N_{1}, N_{2}, \lambda\right)\left(\sum_{|\theta|=\frac{1}{\lambda N_{1}}}\left\|E f_{s_{j}, \theta}\right\|_{L_{a v g}^{4}\left(w_{B_{N_{1}^{2}}}\right)}^{2}\right)^{1 / 2}\left(\sum_{|\theta|=\frac{1}{\lambda N_{1}}}\left\|E f_{s_{j}^{\prime}, \theta}\right\|_{L_{a v g}^{4}\left(w_{B_{N_{1}}}\right)}^{2}\right)^{1 / 2} .
\end{aligned}
$$

Plugging (7.14) into (7.12), and then plugging into (7.11), we derive

$$
\begin{aligned}
&\left.\left\|E f_{1} E f_{2} \mid\right\|_{l^{2}\left(w_{B} N_{1}^{2}\right.}\right) \\
& \lesssim K^{C} \lambda\left(\frac{1}{\lambda}\right)^{1 / 2} \prod_{i=1}^{2}\left(\sum_{|\theta|=\frac{1}{\lambda N_{1}}}\left\|E f_{i, \theta}\right\|_{L_{a v g}^{4}\left(w_{B_{N} 2}\right)}^{2}\right)^{1 / 2} \\
&+\left(\sum_{|\theta|=\frac{N_{2}}{\lambda K N_{1}}}\left\|E f_{i, \theta}\right\|_{L_{a v g}^{4}\left(w_{B_{N_{1}^{2}}}\right)}^{2}\right)^{1 / 2} .
\end{aligned}
$$


Thus we derive

$$
\lambda K\left(N_{1}, N_{2}, \lambda\right) \lesssim K^{C} A\left(N_{1}, N_{2}, \lambda\right)+\lambda K\left(N_{1}, N_{2} / K, \lambda\right)
$$

Thus, Lemma 7.5 follows.

Now we are ready to start with the induction procedure on $N_{2}$. We emphasize again that by Lemma 3.6 we only need to consider the case $\lambda \leq N_{1} / N_{2}$.

7.3.2. First induction: Case $N_{2}^{2} \leq N_{1}$. It will become clear in the following proof why we choose the first splitting point at $N_{1}=N_{2}^{2}$. We start with an estimate for $A\left(\lambda, N_{1}, N_{2}\right)$. We have

Lemma 7.6. When $N_{2} \leq N_{1}^{2}, \lambda \leq N_{1}, \lambda \leq N_{1} / N_{2}$,

$$
A\left(\lambda, N_{1}, N_{2}\right) \lesssim \lambda^{1 / 2} \equiv \lambda \lambda^{-1 / 2}
$$

Assuming Lemma 7.6 for the moment, let us finish the proof of Lemma 3.3 when $N_{1} \geq N_{2}^{2}$. Applying Lemma 7.6 with Lemma 7.5, we derive

$$
K\left(N_{1}, N_{2}, \lambda\right) \lesssim K^{C} \lambda\left(\frac{1}{\lambda}\right)^{1 / 2}+K\left(N_{1}, N_{2} / K, \lambda\right)
$$

when $N_{1} \geq N_{2}^{2}$ and $\lambda \leq N_{1} / N_{2}$. Choosing $1 \ll K \sim N_{2}^{\epsilon^{10}}$, performing induction on $N_{2}$ again, and recalling that the case $N_{2} \lesssim 1$ is covered by Lemma 3.4 then Lemma 3.3 follows when $N_{1} \geq N_{2}^{2}$.

Now, we turn to the proof of Lemma 7.6 .

Proof of Lemma 7.6. Since $N_{1} \leq N_{2}^{2}$, thus $\frac{N_{2}}{N_{1}} \leq \frac{1}{N_{2}}$. (It is exactly because of this that we decided our first splitting point $N_{1} \leq N_{2}^{2}$ ). Thus, the support of $g_{1}, g_{2}$ appearing in (7.9) are (contained in) strips of size $\frac{1}{N_{2}} \times 1$. Thus, in a ball of radius $N_{2}^{2}$, we have

$$
\int\left|E g_{1} E g_{2}\right| w_{B_{N_{2}}} \lesssim \sum_{\left|\theta_{i}\right|=\frac{1}{N_{2}}, \theta_{i} \subset \text { supp } g_{i}} \int\left|E_{g_{1}, \theta_{1}} E g_{2, \theta_{2}}\right| w_{B_{N_{2}^{2}}} .
$$

The proof of (7.19) is essentially the same as the proof of Lemma 5.1] and we leave it to reader.

Note one can use balls $B_{N_{2}^{2}}$ to cover $\tilde{\Omega}:=\left[0, N_{2}^{2}\right] \times\left[0, N_{1} N_{2}\right]^{2}$, (since $\left.N_{1} \geq N_{2}\right)$ thus we extend (7.19) to

$$
\int\left|E g_{1} E g_{2}\right| w_{\tilde{\Omega}} \lesssim \sum_{\left|\theta_{i}\right|=\frac{1}{N_{2}}, \theta_{i} \subset \text { supp } g_{i}} \int\left|E_{g_{1}, \theta_{1}} E g_{2, \theta_{2}}\right| w_{\tilde{\Omega}} .
$$

We claim for any fixed $\theta_{1}, \theta_{2}$, one has

$$
\left\|E g_{1, \theta_{1}} E g_{2, \theta_{2}}\right\|_{L^{2}\left(w_{\tilde{\Omega}}\right)} \lesssim \lambda \lambda^{-1 / 2} \prod_{i=1}^{2}\left(\sum_{\tilde{\theta_{i}} \subset \theta_{i},\left\|\tilde{\theta}_{i}=\frac{1}{\lambda N_{2}}\right\|}\left\|E g_{i, \tilde{\theta}_{i}}\right\|_{L^{4}\left(w_{\tilde{\Omega}}\right)}\right)^{1 / 2}
$$

Plugging (7.21) into (7.20), we have

and the Lemma 7.6 follows.

$$
A\left(N_{1}, N_{2}, \lambda\right) \lesssim \lambda\left(\frac{1}{\lambda}\right)^{1 / 2}
$$

Now we are left with the proof of (7.21). Let $N_{1}^{\prime}=N_{2}, N_{2}^{\prime}=N_{2}^{2} / N_{1} \lesssim 1$. When $N_{1}^{\prime}=N_{2} \leq \lambda$, recall the definition of $\tilde{K}\left(\lambda, N_{1}, N_{2}\right)$ in (3.4) and apply Lemma 3.2, we have

$$
K\left(N_{1}^{\prime}, N_{2}^{\prime}, \lambda\right) \lesssim\left(N_{2}^{\prime}\right)^{\epsilon}\left(\frac{N_{2}^{\prime}}{N_{1}^{\prime}}\right)^{1 / 2} \lesssim\left(\frac{1}{\lambda}+\frac{N_{2}}{N_{1}}\right)^{1 / 2} \lesssim \lambda^{-1 / 2}
$$

The last inequality in (7.23) follows because we always have $\lambda \leq N_{1} / N_{2}$ in the whole induction process. Note (7.23) implies

$$
\begin{aligned}
& \left\|E g_{1, \theta_{1}} E g_{2, \theta_{2}}\right\|_{L^{2}\left(w_{\left[0, N_{2}^{2}\right] \times\left[0, \lambda N_{2}\right]^{2}}\right)} \\
& \lesssim \lambda \tilde{K}\left(N_{1}^{\prime}, N_{2}^{\prime}, \lambda\right) \prod_{i=1}^{2}\left(\sum_{\tilde{\theta_{i}} \subset \theta_{i},\left\|\tilde{\theta}_{i}=\frac{1}{\lambda N_{2}}\right\|}\left\|E g_{i, \tilde{\theta}_{i}}\right\| \|_{L^{4}\left(w_{\left[0, N_{2}^{2}\right] \times\left[0, \lambda N_{2}\right]^{2}}\right)}\right)^{1 / 2} .
\end{aligned}
$$


Since $\lambda \leq N_{1}$, (which is also always the case during the induction process ), $\tilde{\Omega}$ can be covered by the translations of $\left[0, N_{2}^{2}\right] \times\left[0, \lambda N_{2}\right]$, thus (7.24) implies (7.21) by the parallel decoupling Lemma 1.9.

When $\lambda \leq N_{1}^{\prime}$, since $N_{2}^{\prime} \lesssim 1$, by Lemma 3.4, we have

$$
K\left(\lambda, N_{1}^{\prime}, N_{2}^{\prime}\right) \lesssim \lambda^{-1 / 2}
$$

Thus,

$$
\left\|E g_{1, \theta_{1}} E g_{2, \theta_{2}}\right\|_{L^{2}\left(w_{B_{N}}\right)} \lesssim \lambda \lambda^{-1 / 2} \prod_{i=1}^{2}\left(\sum_{\tilde{\theta_{i}} \subset \theta_{i},\left\|\tilde{\theta_{i}}=\frac{1}{\lambda N_{2}}\right\|}\left\|E g_{i, \tilde{\theta}_{i}}\right\|_{L^{4}\left(w_{N_{2}}^{2}\right)}\right)^{1 / 2}
$$

Since one can use $B_{N_{2}^{2}}$ and its translations to $\operatorname{cover} \tilde{\Omega}$, (7.26) implies (7.21) by the parallel decoupling Lemma 1.9

7.3.3. Second induction: Case $N_{2}^{3 / 2} \leq N_{1} \leq N_{2}^{2}$.

Lemma 7.7. When $N_{2}^{3 / 2} \leq N_{1} \leq N_{2}^{2}, \lambda \leq N_{1}$ and $\lambda \leq N_{1} / N_{2}$, we have

$$
A\left(\lambda, N_{1}, N_{2}\right) \lesssim \lambda^{1 / 2} \equiv \lambda \lambda^{-1 / 2}
$$

Clearly, using Lemma 7.5 and arguing as in Section 7.3.2, Lemma 3.3 follows from Lemma 7.7 when $N_{2}^{3 / 2} \leq$ $N_{1} \leq N_{2}^{2}$.

Now we are left with proof of Lemma 7.7, i.e. the estimate (7.27). We will prove that estimate (7.27), in case $N_{2}^{3 / 2} \leq N_{1} \leq N_{2}^{2}$, follows from the fact that Lemma 3.3 holds when $N_{2}^{2} \geq N_{1}$, (given Lemma 3.2).

Proof of Lemma 7.7. The proof starts similarly as the proof of Lemma 7.6. note now we have $N_{2} / N_{1} \geq 1 / N_{2}$. As we derived (7.19), we have in a ball of radius $N_{1}^{2} / N_{2}^{2}$,

$$
\int\left|E g_{1} E g_{2}\right| w_{B}\left(\frac{N_{1}}{\left.N_{2}\right)^{2}}\right) \sum_{\left|\theta_{i}\right|=\frac{N_{2}}{N_{1}}, \theta_{i} \subset \text { supp } g_{i}} \int\left|E_{g_{1}, \theta_{1}} E g_{2, \theta_{2}}\right| w_{B_{\left(N_{1} / N_{2}\right)^{2}}}
$$

Note one can use $B_{\left(\frac{N_{1}}{N_{2}}\right)^{2}}$ and its translations to cover $\tilde{\Omega}$, thus we have

$$
\int\left|E g_{1} E g_{2}\right| w_{\tilde{\Omega}} \lesssim \sum_{\left|\theta_{i}\right|=\frac{N_{2}}{N_{1}}, \theta_{i} \subset \text { supp } g_{i}} \int\left|E_{g_{1}, \theta_{1}} E g_{2, \theta_{2}}\right| w_{\tilde{\Omega}}
$$

The following procedure is essentially the same as in the first induction. Note that to prove (7.27) we only need to further show that for fix $\theta_{1}, \theta_{2}$,

$$
\left\|E g_{1, \theta_{1}} E g_{2, \theta_{2}}\right\|_{L^{2}\left(w_{\tilde{\Omega}}\right)} \lesssim \lambda \lambda^{-1 / 2} \prod_{i=1}^{2}\left(\sum_{\tilde{\theta}_{i} \subset \theta_{i},\left|\tilde{\theta}_{i}\right|=\frac{1}{\lambda N_{2}}}\left\|E g_{i, \tilde{\theta}_{i}}\right\|_{L^{4}\left(w_{\tilde{\Omega}}\right)}\right)^{1 / 2}
$$

where now $\left|\theta_{i}\right|=\frac{N_{2}}{N_{1}}$.

Let $N_{1}^{\prime}=N_{2}, N_{2}^{\prime}=\frac{N_{2}^{2}}{N_{1}}$, note we have $N_{1}^{\prime} \geq\left(N_{2}^{\prime}\right)^{2}$ since $N_{1} \geq N_{2}^{3 / 2}$. When $\lambda \geq N_{1}^{\prime}$, we have by Lemma 3.2

$$
\left\|E g_{1, \theta_{1}} E g_{2, \theta_{2}}\right\|_{L^{2}\left(w_{\left[0, N_{2}^{2}\right] \times\left[0, \lambda N_{2}\right]^{2}}\right)} \lesssim \lambda\left(\frac{N_{2}^{\prime}}{N_{1}^{\prime}}\right)^{-1 / 2} \prod_{i=1}^{2}\left(\sum_{\tilde{\theta}_{i} \subset \theta_{i},\left|\tilde{\theta}_{i}\right|=\frac{1}{\lambda N_{2}}}\left\|E g_{i, \tilde{\theta}_{i}}\right\|_{L^{4}\left(w_{\left[0, N_{2}\right] \times\left[0, \lambda N_{2}\right]^{2}}\right)}\right)^{1 / 2} .
$$

Since one can use $\left[0, N_{2}^{2}\right] \times\left[0, \lambda N_{2}\right]^{2}$ to cover $\tilde{\Omega}$, (7.30) follows from (7.31), (note $\frac{N_{2}^{\prime}}{N_{1}^{\prime}}=\frac{N_{2}}{N_{1}} \leq \lambda^{-1}$ ).

When $\lambda \leq N_{1}^{\prime}$, since one can use $B_{N_{2}^{2}}$ to cover $\tilde{\Omega}$, to prove (7.30), we need only to show

$$
\left\|E g_{1, \theta_{1}} E g_{2, \theta_{2}}\right\|_{L^{2}\left(w_{B_{2}}^{2}\right)} \lesssim \lambda \lambda^{-1 / 2} \prod_{i=1}^{2}\left(\sum_{\tilde{\theta_{i}} \subset \theta_{i},\left\|\tilde{\theta_{i}}=\frac{1}{\lambda N_{2}}\right\|}\left\|E g_{i, \tilde{\theta_{i}}}\right\|_{L^{4}\left(w_{B_{N_{2}^{2}}}\right)}\right)^{1 / 2}
$$

which is equivalent to $K\left(N_{1}^{\prime}, N_{2}^{\prime}, \lambda\right) \leq \frac{1}{\lambda}$. But recall that $N_{1}^{\prime} \geq\left(N_{2}^{\prime}\right)^{2}$, thus this is exactly what we proved in first induction, i.e. Lemma 3.3 holds when $N_{1} \geq N_{2}^{2}$. 
7.3.4. Later inductions and the conclusion of the induction process. Recall that the first induction covers the case $N_{1} \geq N_{2}^{2}$ and the second inductions covers the case $N_{2}^{\alpha} \leq N_{1} \leq N_{2}^{2}, \alpha=3 / 2$. The goal now is to use induction to cover the case $N_{2}^{\alpha} \leq N_{1}$, all the way to $\alpha=1$. The arguments here are similar to those for the second induction presented in Section 7.3.3. Let $N_{1}^{\prime}=N_{2}, N_{2}^{\prime}=N_{2}^{2} / N_{1}$, then $N_{1}^{\prime} \geq\left(N_{2}^{\prime}\right)^{\alpha}$ is equivalent to $N_{1} \geq N_{2}^{\frac{2 \alpha-1}{\alpha}}$. Once we show that Lemma 3.3 holds when $N_{2}^{\alpha} \leq N_{1} \leq N_{2}^{2}$, we would be able to extend Lemma 7.7 to the case when $N_{2}^{\frac{2 \alpha-1}{\alpha}} \leq N_{1}$, which in turn proves that Lemma 3.3 holds when $N_{2}^{\frac{2 \alpha-1}{\alpha}} \leq N_{1} \leq N_{2}^{2}$. The induction would not end until $\alpha=1$. We finally point out, that only an induction with finite steps is involved.

To show Lemma 3.3 for a fixed $\epsilon_{0}$, we may pick an $\tilde{\epsilon} \ll \epsilon_{0}$, then we perform the induction for $\tilde{\epsilon}$ as above.

After we prove Lemma 3.3 for $N_{1} \geq N_{2}^{1+\tilde{\epsilon}}$, we are left with the case $N_{1} \leq N_{2}^{1+\tilde{\epsilon}}$. We first use Hölder inequality to shrink the size of the cap from $N_{2} / N_{1}$ to $N_{2}^{1-2 \tilde{\epsilon}} / N_{1}$, which only gives a loss of $N_{2}^{C \tilde{\epsilon}} \ll N_{2}^{\epsilon_{0}}$. Then we use Lemma (3.3) in the case $N_{1} \geq N_{2}^{1+\tilde{\epsilon}}$ again.

Thus, Lemma 3.3 holds for all the case for our fixed $\epsilon_{0}$.

7.4. The high dimension case. To handle the case $d \geq 3$, we are left with the proof of Lemma 3.5. The proof is indeed similar to previous arguments in this section and easier. The proof relies on the linear decoupling estimate in [4].

As aforementioned, applying Lemma 5.1, taking $v=N_{2} / N_{1}$ and $R=N_{1}^{2}$, we can decouple the $\frac{N_{2}}{N_{1}}$ caps into $\left(\frac{N_{2}}{N_{1}}, \frac{N_{2}^{2}}{N_{1}^{2}}\right)$ plates without any loss, i.e. (7.1). However, since we are in the case $\lambda \leq N_{1} / N_{2}^{2}$, indeed $N_{2}^{2} / N_{1}^{2} \lesssim \frac{1}{\lambda N_{1}}$, we only need a weaker version of (7.1), i.e. we only want to decouple the $\frac{N_{2}}{N_{1}}$ caps into $\left(\frac{N_{2}}{N_{1}}, \frac{1}{\lambda N_{1}}\right)$ plates:

$$
\int\left|E f_{1} E f_{2}\right|^{2} w_{B_{N_{1}^{2}}} \lesssim \sum_{\tau_{1}, \tau_{2}} \int\left|E f_{1, \tau_{1}} E f_{2, \tau_{2}}\right|^{2} w_{B_{N_{1}}^{2}}
$$

Here $\tau_{i}$ are $\left(\frac{N_{2}}{N_{1}}, \frac{1}{\lambda N_{1}}\right)$ plates as described in Lemma 5.1. Note (7.33) follows from (7.1).

Now, for each $\tau_{i}$ fixed, we further decouple $\tau_{i}$ into $\left(\frac{1}{N_{1}}, \frac{1}{\lambda N_{1}}\right)$ plates via linear decoupling in [4, here recalled in (1.11). Note direct application of linear decoupling in dimension $d$ gives us

$$
\left\|E f_{\tau_{i}}\right\|_{L^{4}\left(w_{B_{N}}\right)} \lesssim N_{2}^{\epsilon}\left(N_{2}^{2}\right)^{\frac{d}{4}-\frac{d+2}{8}}\left(\sum_{v_{i} \subset \tau_{i}}\left\|E f_{v_{i}}\right\|_{L^{4}\left(w_{B_{N_{1}^{2}}}\right)}^{2}\right)^{1 / 2}
$$

However, we are able to use (1.11) when the dimension is $d-1$ rather than $d$, because our plates are so thin (of scale $\frac{1}{\lambda N_{1}} \leq \frac{1}{N_{1}}$ ), which reduce the dimension by 1. Indeed, Linear decoupling (1.11) not only work for those functions which are exactly supported in parabola $P$ but also those which are supported in a $N_{1}^{-2}$ neighborhood of $P$. This is consistent in uncertainty principle, since in physical space we of scale $N_{1}^{2}$, in frequency space any scale of $N_{1}^{-2}$ cannot be differentiated. Since our plate are so thin, of scale $\frac{1}{\lambda N_{1}} \leq N_{1}^{-2}$, one could indeed view it as a $N_{1}^{-2}$ neighborhood of some $d-1$ dimensional parabola. To be more specific and use $\tau_{2}$ as example, since $\tau_{2}$ is supported at the origin. Let $\pi_{t}^{-1}\left(\tau_{2}\right)$ be the pull back image of $\tau_{2}$ to the paraboloid. The fourier inverse transform of $E f_{\tau_{2}}$ is supported on $\pi_{t}^{-1}\left(\tau_{2}\right)$. One can see that if we project along $x_{1}$-axis, the projection image of $\pi_{t}^{-1}\left(\tau_{2}\right)$ is the $\left(\frac{1}{\lambda N_{1}}\right)^{2}$-neighborhood of a $(d-1)$-dimensional paraboloid (a piece of length $\frac{N_{2}}{N_{1}}$ ).

Now, apply $d-1$ dimensional linear decoupling, we improve (7.34) into

$$
\left\|E f_{\tau_{i}}\right\|_{L^{4}\left(w_{B_{N_{1}^{2}}}\right)} \lesssim N_{2}^{\epsilon}\left(N_{2}^{2}\right)^{\frac{d-1}{4}-\frac{d+1}{8}}\left(\sum_{v_{i} \subset \tau_{i}}\left\|E f_{v_{i}}\right\|_{L^{4}\left(w_{B_{N_{1}^{2}}}\right)}^{2}\right)^{1 / 2},
$$

where $v_{i}$ are $\left(\frac{1}{N_{1}}, \frac{1}{\lambda N_{1}}\right)$ plates.

Finally, similarly to the derivation of (6.4), we decouple $v_{i}$ into caps of radius $\frac{1}{\lambda N_{1}}$,

$$
\left\|E f_{v_{i}}\right\|_{L^{4}\left(w_{B_{N}}^{2}\right)}^{4} \lesssim \lambda^{(d-1)}\left(\sum_{\theta_{i} \subset v_{i}}\left\|E f_{\theta_{i}}\right\|_{L^{4}\left(w_{B_{N_{1}^{2}}}\right)}^{2}\right)^{2}
$$

We remark that each $v_{i}$ can be coved by $\lambda^{d-1}$ rather than $\lambda^{d}$ caps of radius $\frac{1}{\lambda N_{1}}$. Plugging (7.36) into (7.35), then plugging it into (17.33), we derive

$$
\left\|E f_{1} E f_{2}\right\|_{L_{a v g}^{2}\left(w_{B_{1}}^{2}\right)} \leq \lambda^{d-1 / 2} N_{2}^{\frac{d-3}{2}} \prod_{j=1}^{2}\left(\sum_{|\theta|=\frac{1}{\lambda N_{1}}}\left\|E f_{j, \theta}\right\|_{L_{a v g}^{4}\left(w_{B_{1}}^{2}\right)}^{2}\right)^{1 / 2} .
$$


Thus, the desired estimate for $K\left(\lambda, N_{1}, N_{2}\right)$ follows.

\section{Appendix A. Sharpness of Theorem 1.3 and Theorem 1.1}

The sharpness (up to $N_{2}^{\epsilon}$ ) of Theorem 1.3 is provided by the following examples. One can also re-scale those example to show the sharpness of Theorem 1.1 .

We take $E f_{1}=\sum_{\xi \in \Lambda_{\lambda N_{1}},|\xi| \leq \frac{N_{2}}{N_{1}}} e^{2 \pi i\left(\xi \cdot x+|\xi|^{2} t\right)}$ and $f_{2}=f_{1}(\cdot-(1,0, \ldots, 0))$. Then $\left|E f_{1}\right|$ is about $\left(\lambda N_{2}\right)^{d}$ at $B\left(0, \frac{N_{1}}{N_{2}}\right)$ in $\mathbb{R}^{d+1}$. Note that it follows from uncertainty principle, it is locally constant in any ball of size $\frac{N_{1}}{N_{2}}$ and one can easily compute $\left|E f_{1}(0)\right| \sim\left(\lambda N_{2}\right)^{d}$. Also note $\left|E f_{1}\right|$ has periodicity around $\lambda N_{1}$ in all components of $x$, (not necessarily in $t)$. The same is true for $\left|E f_{2}\right|$. Thus,

$$
\begin{aligned}
\left\|E f_{1} E f_{2}\right\|_{L^{2}\left(w_{\Omega}\right)}^{2} & \gtrsim\left(\lambda N_{2}\right)^{4 d}\left|B\left(0, \frac{N_{1}}{N_{2}}\right)\right|\left(\lambda N_{1}\right)^{d} \\
& \gtrsim \lambda^{5 d} N_{1}^{2 d+1} N_{2}^{3 d-1}
\end{aligned}
$$

Each cap $\theta_{j}$ of radius $\frac{1}{\lambda N_{1}}$ contains at most one point $\xi \in \Lambda_{\lambda N_{1}}$. Hence $\left\|E f_{j, \theta_{j}}\right\|_{L^{4}\left(w_{\Omega}\right)}^{4} \lesssim|\Omega|=N_{1}^{2}\left(\lambda N_{1}\right)^{2 d}$.

$$
\begin{aligned}
\Pi_{j=1}^{2}\left(\sum_{\left|\theta_{j}\right|=\frac{1}{\lambda N_{1}}}\left\|E f_{j, \theta_{j}}\right\|_{L^{4}\left(w_{\Omega}\right)}^{2}\right) & \lesssim\left(\lambda N_{2}\right)^{2 d} N_{1}^{2}\left(\lambda N_{1}\right)^{2 d} \\
& \lesssim \lambda^{4 d} N_{1}^{2 d+2} N_{2}^{2 d}
\end{aligned}
$$

This example shows that the term with $\frac{N_{2}^{d-1}}{N_{1}}$ is sharp for both $d=2$ and $d \geq 3$.

When $d=2$, we consider the example when

$$
\begin{aligned}
& E f_{1}=\sum_{\xi \in \Lambda_{\lambda N_{1}}, \xi_{1}=1,\left|\xi_{2}\right| \leq \frac{1}{N_{1}}} e^{2 \pi i\left(\xi \cdot x+|\xi|^{2} t\right)} \\
& E f_{2}=\sum_{\xi \in \Lambda_{\lambda N_{1}}, \xi_{1}=0,\left|\xi_{2}\right| \leq \frac{1}{N_{1}}} e^{2 \pi i\left(\xi \cdot x+|\xi|^{2} t\right)} .
\end{aligned}
$$

$\left|E f_{1}\right|$ is about $\lambda$ in the box of height $N_{1}^{2}$ (i.e. the $t$ direction), width $N_{1}$, (i.e the $x_{2}$ direction) and length $\left(\lambda N_{1}\right)^{2}$, (i.e. the $x_{1}$ direction) centered at origin. $\left|E f_{2}\right|$ is the same size in the same box. Moreover, $E f_{1}$ and $E f_{2}$ both have periodicity around $\lambda N_{1}$ in $x_{2}$.

$$
\begin{aligned}
\left\|E f_{1} E f_{2}\right\|_{L^{2}\left(w_{\Omega}\right)}^{2} & \gtrsim \lambda^{4} N_{1}^{2} \cdot N_{1} \cdot\left(\lambda N_{1}\right)^{2} \cdot \lambda N_{1} \\
& \gtrsim \lambda^{7} N_{1}^{6}
\end{aligned}
$$

As calculated previously, $\left\|E f_{j, \theta_{j}}\right\|_{L^{4}\left(w_{\Omega}\right)}^{4}=|\Omega|$.

$$
\begin{aligned}
\Pi_{j=1}^{2}\left(\sum_{\left|\theta_{j}\right|=\frac{1}{\lambda N_{1}}}\left\|E f_{j, \theta_{j}}\right\|_{L^{4}\left(w_{\Omega}\right)}^{2}\right) & \lesssim \lambda^{2} \cdot|\Omega| \\
& \lesssim \lambda^{6} N_{1}^{6}
\end{aligned}
$$

This example shows that when $d=2$, the term with $\frac{1}{\lambda}$ is sharp.

When $d \geq 3$, we consider the example when

$$
\begin{aligned}
& E f_{1}=\sum_{\xi \in \Lambda_{\lambda N_{1}}, \xi_{1}=1,\left|\left(\xi_{2}, \ldots, \xi_{d}\right)\right| \leq \frac{N_{2}}{N_{1}}} e^{2 \pi i\left(\xi \cdot x+|\xi|^{2} t\right)} \\
& E f_{2}=\sum_{\xi \in \Lambda_{\lambda N_{1}}, \xi_{1}=0,\left|\left(\xi_{2}, \ldots, \xi_{d}\right)\right| \leq \frac{N_{2}}{N_{1}}} e^{2 \pi i\left(\xi \cdot x+|\xi|^{2} t\right)} .
\end{aligned}
$$

Notice that we construct the example in $d \geq 3$ differently, the support of $f_{j}$ is in a thin plate of radius $\frac{N_{2}}{N_{1}}$ instead of the $\frac{1}{N_{1}}$ as in 2-dimensional example.

$\left|E f_{1}\right|$ is about $\left(\lambda N_{2}\right)^{d-1}$ in a box of size $\left(\frac{N_{1}}{N_{2}}\right) \times \cdots \times \frac{N_{1}}{N_{2}} \times\left(\frac{N_{1}}{N_{2}}\right)^{2} \times\left(\lambda N_{1}\right)^{2} \cdot\left|E f_{2}\right|$ is about $\left(\lambda N_{2}\right)^{d-1}$ in the same box. Both $E f_{1}$ and $E f_{2}$ has periodicity around $\lambda N_{1}$ in $x_{2}, \ldots, x_{d}$-directions. 


$$
\begin{aligned}
\left\|E f_{1} E f_{2}\right\|_{L^{2}\left(w_{\Omega}\right)}^{2} & \gtrsim\left(\lambda N_{1}\right)^{4(d-1)}\left(\frac{N_{1}}{N_{2}}\right)^{d+1}\left(\lambda N_{1}\right)^{2}\left(\lambda N_{1}\right)^{d-1} \\
& \gtrsim \lambda^{5 d-3} N_{1}^{2 d+2} N_{2}^{3 d-5} \\
\Pi_{j=1}^{2}\left(\sum_{\left|\theta_{j}\right|=\frac{1}{\lambda N_{1}}}\left\|E f_{j, \theta_{j}}\right\|_{L^{4}\left(w_{\Omega}\right)}^{2}\right) & \lesssim\left(\lambda N_{2}\right)^{2(d-1)} \cdot|\Omega| \\
& \lesssim \lambda^{4 d-2} N_{1}^{2 d+2} N_{2}^{2 d-2}
\end{aligned}
$$

This example shows that when $d \geq 3$, the term with $\frac{N_{2}^{d-3}}{\lambda}$ is sharp.

\section{REFERENCES}

[1] J. Bourgain. Fourier transform restriction phenomena for certain lattice subsets and applications to nonlinear evolution equations. Geometric and Functional Analysis, 3(3):209-262, 1993.

[2] J. Bourgain. Refinements of Strichartz inequality and applications to 2D-NLS with critical nonlinearity. International Mathematics Research Notices, 1998(5):253-283, 1998.

[3] J. Bourgain. Moment inequalities for trigonometric polynomials with spectrum in curved hypersurfaces. Israel Journal of Mathematics, 193(1):441-458, 2013.

[4] J. Bourgain and C. Demeter. The proof of the $L^{2}$ decoupling conjecture. Annals of mathematics, 182(1):351-389, 2015.

[5] J. Bourgain and C. Demeter. A study guide for the $L^{2}$ decoupling theorem. arXiv preprint arXiv:1604.06032, 2016.

[6] J. Colliander, M. Keel, G. Staffilani, H. Takaoka, and T. Tao. Almost conservation laws and global rough solutions to a nonlinear Schrödinger equation. Mathematical Research Letters, 9(5), 2002.

[7] D. De Silva, N. Pavlovic, G. Staffilani, and N. Tzirakis. Global well-posedness for a periodic nonlinear schrodinger equation in 1d and 2d. DYNAMICAL SYSTEMS, 19(1):37-65, 2007.

[8] Y. Deng and P. Germain. Growth of solution of NLS on irrational tori. Private communication.

[9] Y. Deng, P. Germain, and L. Guth. Strichartz estimates on irrational tori. Private communication.

[10] Z. Guo, T. Oh, and Y. Wang. Strichartz estimates for Schrödinger equations on irrational tori. Proceedings of the London Mathematical Society, page pdu025, 2014.

[11] Z. Hani. A bilinear oscillatory integral estimate and bilinear refinements to Strichartz estimates on closed manifolds. Analysis \& PDE, 5(2):339-363, 2012.

[12] S. Herr, D. Tataru, N. Tzvetkov, et al. Global well-posedness of the energy-critical nonlinear Schrödinger equation with small initial data in $H^{1}\left(\mathbb{T}^{3}\right)$. Duke Mathematical Journal, 159(2):329-349, 2011.

[13] A. D. Ionescu, B. Pausader, et al. The energy-critical defocusing NLS on $\mathbb{T}^{3}$. Duke Mathematical Journal, 161(8):1581-1612, 2012.

[14] R. Killip and M. Visan. Scale-invariant strichartz estimates on tori and applications. Mathematical Research Letters, 23(2):445-472, 2016.

[15] J. Ramos. The trilinear restriction estimate with sharp dependence on the transversality. arXiv preprint arXiv:1601.05750, 2016. 